\title{
A locus coeruleus-norepinephrine account of individual differences in working memory capacity and attention control
}

\author{
Nash Unsworth $^{1}$ - Matthew K. Robison ${ }^{1}$
}

Published online: 20 January 2017

(C) Psychonomic Society, Inc. 2017

\begin{abstract}
Studies examining individual differences in working memory capacity (WMC) have suggested that low WMC individuals have particular deficits in attention control processes compared to high WMC individuals. In the current article we suggest that part of the WMC-attention control relation is due to variation in the functioning of the locus coeruleus-norepinephrine system (LC-NE). Specifically, we suggest that because of dysregulation of LC-NE functioning, the fronto-parietal control network for low WMC individuals is only weakly activated, resulting in greater default-mode network activity (and greater mind-wandering) for low WMC individuals compared to high WMC individuals. This results in disrupted attention control and overall more erratic performance (more lapses of attention) for low WMC individuals than for high WMC individuals. This framework is used to examine previous studies of individual differences in WMC and attention control, and new evidence is examined on the basis of predictions of the framework to pupillary responses as an indirect marker of LC-NE functioning.
\end{abstract}

Keywords Working memory · Individual differences ·

Attention control

Working memory, our ability to actively maintain, manipulate, and retrieve task-relevant information, is a core cognitive construct that is needed in a host of activities. Indeed, individual differences in working memory capacity (WMC) have long

Nash Unsworth

nashu@uoregon.edu

1 Department of Psychology, University of Oregon, Eugene, OR 97403, USA been shown to be powerful predictors of performance in a number of domains. For example, research has found that WMC predicts performance on a number of measures of higher-order cognition including fluid reasoning (Engle et al., 1999; Kane et al., 2004; Kyllonen \& Christal, 1990), reading comprehension (Daneman \& Carpenter, 1980), learning (Kyllonen \& Stephens, 1990; Unsworth \& Engle, 2005), and standardized tests of scholastic aptitude (Engle et al., 1999). Furthermore, measures of WMC have been shown to predict important phenomena such as early-onset Alzheimer disease (Rosen, Bergeson, Putnam, Harwell, \& Sunderland, 2002), life-event stress (Klein \& Boals, 2001), aspects of personality (Unsworth, Miller, Lakey, Young, Meeks \& Campbell, 2009), susceptibility to choking under pressure (Beilock \& Carr, 2005), and stereotype threat (Schamader \& Johns, 2003). Clearly WMC is related to a number of important abilities, yet the reasons for these relations are still not fully understood. Recently we have suggested a multifaceted view of individual differences in WMC in which individual differences are driven by multiple different facets (Unsworth, 2016; Unsworth et al., 2014). These facets include capacity of primary memory, attention control, and secondary memory abilities. This work builds on prior research suggesting that individual differences in attention control (or executive attention) are one key to understanding the explanatory power of WMC (Engle \& Kane, 2004; Kane \& Engle, 2002; Unsworth \& Engle, 2007). In the current article we extend the attention control (or executive attention) view of WMC by suggesting that individual differences in WMC and attention control are largely driven by differences in fluctuations of attention control regulated by the locus coeruleus-norepinephrine system. Specifically, we utilize Cronbach (1957) and others' (Cohen, 1994; Underwood, 1975) call to analyses of individual differences (along with experimental methods) in order to gain a better understanding of the underlying processes and potential neural 
mechanisms. For the most part we focus on the relation between attention control and WMC, but briefly examine implications for both capacity and secondary memory abilities. Below, we review investigations into individual differences linking WMC and attention control, and present a general framework within which to interpret individual differences in WMC and attention control. Finally, we present evidence from pupillometry studies supporting such a view.

\section{Working memory capacity and attention control}

The notion that working memory and attention are strongly linked has been a basic component of models of working memory since their inception. Indeed, Baddeley (1993) noted that in many situations working memory could really be thought of as working attention. In terms of individual differences in WMC work by Engle, Kane, Conway and colleagues (Engle \& Kane, 2004; Kane \& Engle, 2002; Kane et al., 2007) has strongly suggested that differences in WMC are partially a result of differences in attention control. By attention control we mean the set of processes that allow us to focus selectively and actively maintain task-relevant information in the presence of internally or externally distracting information. In particular, this view suggests that individuals high in WMC are better at controlling aspects of their attention to actively maintain goal-relevant information in order to successfully perform a task than are individuals low in WMC. Furthermore, these differences are especially pronounced under conditions of high interference or distraction in which attentional capture away from task- or goal-relevant information is likely (e.g., Engle \& Kane, 2004). Thus, high WMC individuals are better at preventing interference or distraction than low-WMC individuals, and this attention control ability is needed in a host of activities regardless of specific stimulus or processing domains. By this account individual differences in WMC are partially determined by individual differences in attention control that manifest themselves not only on various attention control tasks (see below), but also on various working memory measures. Indeed, recent research suggests that individual differences in working memory are partially due to lapses of attention that occur on various working memory tasks (Adam et al., 2015; Mrazek et al., 2012; Unsworth \& Robison, 2015, 2016a). Below we review evidence suggesting WMC differences in terms of (1) performance on various attention control tasks, (2) attentional failures in and out of the laboratory, and (3) consistency of attentional control.

\section{Working memory capacity differences on attention con-} trol tasks: Much of the evidence in favor of an attention control view of WMC comes from extreme groups and factor analytic studies demonstrating relations between measures of WMC and attention control. For example, WMC differences have been demonstrated in dichotic listening (Colflesh \& Conway, 2007; Conway, Cowan, \& Bunting, 2001), Stroop interference (Hutchison, 2011; Kane \& Engle, 2003; Long \& Prat, 2002; Meier \& Kane, 2013; Morey et al., 2012), flanker interference (Heitz \& Engle, 2007; Redick \& Engle, 2006), performance on the antisaccade task (Kane et al., 2001; Unsworth, Schrock, \& Engle, 2004), performance on the psychomotor vigilance task (Unsworth et al., 2010; Unsworth \& Spillers, 2010), performance on the Sustained Attention to Response Task (SART; McVay \& Kane, 2009), performance on versions of go/no-go tasks (Redick et al., 2011), performance on the AX-CPT task (Redick, 2014; Redick \& Engle, 2011; Richmond, Redick, \& Braver, 2015), performance on cued visual search tasks (Poole \& Kane, 2009), and performance on some versions of the Simon task (Meier \& Kane, 2015; Weldon et al., 2013; but see Keye et al., 2009). Clearly, individual differences in WMC are related to performance on a number of attention control tasks. Furthermore, these differences are found when examining latent variables composed of the shared variance among multiple attention control tasks. For example, Unsworth and Spillers (2010) had participants perform a number of WMC tasks as well as antisaccade, flankers, Stroop, and the psychomotor vigilance task. It was found that all of the attention control tasks loaded on the same attention control factor and this factor was strongly related to a latent WMC factor (see also Kane et al., 2016; McVay \& Kane, 2012; Unsworth, Fukuda, Awh, \& Vogel, 2014; Unsworth \& McMillan, 2014). Indeed, as shown in Fig. 1, examining data from 646 participants pooled from a number of published studies from our laboratory suggested that performance on antisaccade, flankers, and the psychomotor vigilance task all loaded onto the same latent attention control

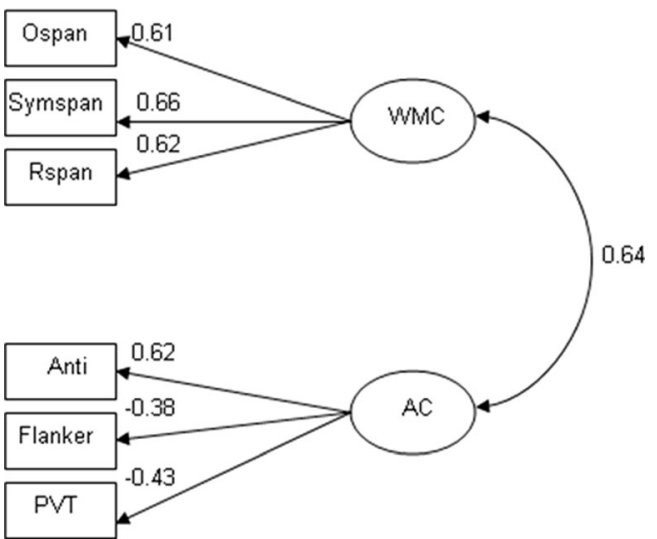

Fig. 1 Confirmatory factor analysis examining relation between working memory capacity (WMC) and attention control (AC). All paths are significant at the $\mathrm{p}<.05$ level. The fit of the model was acceptable, $\chi^{2}$ $(7)=11.29, p=.12, \mathrm{RMSEA}=.03, \mathrm{NNFI}=.99, \mathrm{CFI}=.99, \mathrm{SRMR}=.02$. Furthermore, the two-factor model shown fitted significantly better than a one-factor model, $\Delta \chi^{2}(1)=33.95, p<.001$. Ospan operation span recall, Rspan reading span recall, Symspan symmetry span recall, Anti antisaccade accuracy, Flanker Flanker interference score, $P V T$ slowest $20 \%$ of trials on the psychomotor vigilance task 
factor, and this factor was strongly correlated with WMC. Thus, WMC is related to attention control at both the task and latent levels.

Furthermore, it should be noted that our attention control construct is conceptually similar to Miyake and Friedman's inhibition factor (Friedman \& Miyake, 2004; Friedman et al., 2006, 2008; Miyake et al., 2000; Miyake \& Friedman, 2012). In particular, both are made up of similar tasks (e.g., antisaccade, Stroop, flankers, etc.) and both are strongly related to working memory factors. Additionally, along with other executive functions there is a strong genetic component to the inhibition factor and it is related to a number of clinically and societally important behaviors (Friedman et al., 2008; Miyake \& Friedman, 2012; see also Kane et al., 2016). Thus, when talking about the attention control construct it is important to keep in mind that it is very similar to the inhibition construct in Miyake and Friedman's work.

Collectively these results are in line with an attention control view of WMC, which suggests that individual differences in WMC are partially due to differences in the ability to actively maintain task goals in the presence of distraction and interference. That is, top-down attention control processes are needed to maintain task goals and bias responding so that the appropriate behavior is executed. In many situations, participants will have to actively maintain a novel task goal that is in direct opposition to prepotent response tendencies (Roberts \& Pennington, 1996). If there is a failure of active goal maintenance, then it is likely that prepotent response tendencies will guide behavior leading to the execution of the incorrect response and overall goal neglect (Duncan, 1995). That is, in situations when attention is tightly focused on the task goal, performance will be both fast and accurate. However, if attention is not tightly focused on the task goal, goal neglect can occur, which will lead to overall slower responses or to very fast errors that are guided by prepotent tendencies. In conditions where active maintenance of task goals is not required for correct responding, WMC differences typically do not occur. That is, there are boundary conditions regarding the relation between WMC and various attention control processes (Kane et al., 2006; Meier \& Kane, 2013, 2015; Morey et al., 2012; Poole \& Kane, 2009; Unsworth et al., 2012). It is not the case that WMC is related to all manifestations of attention control, but rather that particular aspects of attention control are related to WMC. Thus, there are clear relations between individual differences in $\mathrm{WMC}$ and performance on a variety of attention control tasks and these relations seem to mostly be due to differences in the ability to actively maintain task goals and prevent goal neglect and attentional capture.

\section{Working memory capacity and attentional failures in and} out of the laboratory: In addition to demonstrating relations between WMC and performance on a variety of attention control tasks, recent work has suggested relations between
WMC and subjective attentional failures both in and out of the laboratory. For example, Kane et al. (2007) had participants perform a number of WMC tasks in the laboratory and then participants carried PDAs (personal digital assistants) for a week. Periodically throughout the day the PDAs would beep and participants would have to answer a variety of questions about whether they had just been mind-wandering. Importantly, Kane et al. found that low WMC individuals experienced more mind-wandering in daily life when their current task required concentration, was challenging, or was effortful. Thus, low WMC individuals experienced more realworld attentional failures in situations that required a great deal of control. Other factors such as boredom or sleepiness did not moderate the relation between WMC and mind-wandering. In line with attention control views this suggests that low WMC individuals found it more difficult than high WMC individuals to sustain their attention on challenging and demanding tasks leading to attention failures (i.e., more mindwandering). However, on tasks that did not require a great deal of effort, WMC was unrelated to mind-wandering.

In a similar vein, Unsworth, Brewer, and Spillers (2012) had participants perform a number of tasks in the laboratory (WMC, attention control, prospective memory, retrospective memory) and then carry a diary around for a week logging their various cognitive failures. Important for the current discussion, Unsworth et al. found that WMC and attention control assessed in the laboratory predicted everyday attentional failures. In particular, low WMC and low attention control individuals reported more external distraction, more absentmindedness, and more mind-wandering than high WMC individuals. In a subsequent analysis of the data focusing only on the attentional failures, Unsworth, McMillan, Brewer, and Spillers (2012) found that most attention failures occurred either in the classroom or while studying. Like Kane et al. (2007), Unsworth et al. found that WMC and attention control predicted everyday attentional failures that seemed to require a high degree of focused and sustained attention, but did not predict all types of attentional failures. In particular, WMC and attention control were related to being distracted, while studying, mind-wandering in class, and being distracted in class and these three types of attentional failures loaded onto the same latent factor. Importantly this latent attentional failure factor was significantly related to both WMC and attention control. Thus, WMC strongly predicts individual differences in attention control in and out of the laboratory.

In addition to assessing attentional failures in everyday settings, recent research has focused on examining subjective attentional failures during the laboratory-based attention control tasks and whether WMC predicts the occurrence of these attentional failures. For example, McVay and Kane (2009) utilized thought probe techniques in which periodically throughout a task participants are probed with regard to their current state (on-task or off-task). Prior work with these 
techniques has found that participants report mind-wandering during many cognitive tasks and that the degree of mindwandering varies as a function of task variables such as time on task, task complexity, and task difficulty (McVay \& Kane, 2010; Smallwood \& Schooler, 2006). Importantly, mindwandering rates correlate with task performance such that performance is lower when participants report that they were mind-wandering on the preceding trial compared to when participants report that they are currently focused on the task (McVay \& Kane, 2010; Smallwood \& Schooler, 2006). Furthermore, mind-wandering rates in the laboratory predict mind-wandering rates in everyday life (McVay, Kane, \& Kwapil, 2009). Using these thought probe techniques, McVay and Kane (2009) found that low WMC individuals reported more mind-wandering during the sustained attention to response task (SART) than high WMC individuals, and importantly that mind-wandering rates partially mediated the relation between WMC and performance on the SART. McVay and Kane interpreted their results as being consistent with attention control views of WMC in that low WMC individuals were more likely to experience goal neglect than high WMC individuals due to their attention being hijacked by internal distracting thoughts (mind-wandering). Thus, low WMC individuals were more likely to respond habitually because their attention was focused internally rather than externally on the task.

Subsequent work by McVay and Kane (2012a; Kane \& McVay, 2012; Kane et al., 2016) has found that mindwandering rates across various tasks (Stroop, reading comprehension) correlate quite well and load on the same latent factor. Importantly, this latent mind-wandering factor correlates well with latent WMC and attention control factors (see also Unsworth \& McMillan, 2013). Additionally, recent research has shown that mind-wandering even occurs during WMC tasks (Mrazek et al., 2012; Unsworth \& Robison, 2016a). Similarly, Unsworth and McMillan (2014) had participants perform a number of WMC and attention control tasks in which we probed participants about their current attentional state during the attention tasks. Like McVay and Kane (2012a) we asked if participants were thinking about the current task or mind-wandering. In addition we also asked if participants were distracted by information in the external environment (Stawarczyk et al., 2011). The idea being that WMC and low attention control individuals will be more likely than high WMC individuals to have their attention captured by both internal distractors (mind-wandering) and potent external distractors (see also Robison \& Unsworth, 2015). Much like the flashing cue in the antisaccade task, low WMC individuals should be more likely than high WMC individuals to be captured by irrelevant sensory information (such as loud noises or flickering lights) while trying to sustain their attention on the task at hand. Unsworth and McMillan (2014a) found that mind-wandering and external distraction were correlated at the latent level (see also Unsworth et al., 2012 for a similar demonstration in everyday attention failures) and both were correlated with WMC and attention control. In fact, the shared variance among external distraction, mind-wandering, and performance on the attention control tasks was strongly correlated with WMC. Consistent with attention control views, when attention is tightly focused on the task, performance will be good. However, if attention is not tightly focused on the task, goal neglect can occur which can lead to a hijacking of attention to irrelevant internal or external sources. Note prior research has suggested that mind-wandering can be either deliberate (intentional) or spontaneous (unintentional; Grodsky \& Giambra, 1990, 1991; Seli et al., 2016) In situations where attention needs to be allocated to external information for accurate task performance (like on the various attention control tasks discussed throughout), it is likely that the hijacking of attention to internal sources reflects spontaneous (unintentional) mind-wandering rather than a deliberate (intentional) withdrawal of resources away from the primary task. Future research is needed to better examine whether the relations among WMC, attention control, and mindwandering reflect spontaneous or deliberate mind-wandering. Overall these results are consistent with the notion that WMC is related to subjective attentional state and attentional failures in and out of the laboratory.

\section{Working memory capacity and consistency of attention} control: Prior work clearly shows a relation between WMC and attention control. Despite initial evidence suggesting a strong link between WMC and attention control, the reason for this relation is not known. Early research suggested that this relation was due to the fact that high WMC individuals have more attention control than low WMC individuals akin to classical conceptions of capacity limits (e.g., Conway \& Engle, 1996; but see Conway, Cowan, \& Bunting, 2001; Conway \& Kane, 2001). That is, high WMC individuals have more capacity than low WMC individuals, which allows them greater control in a variety of situations. This view suggests that when attention control is required low WMC individuals should always perform more poorly than high WMC individuals. Prior research has largely corroborated this view by examining mean levels of performance between high and low WMC individuals on a variety of tasks. Another possibility is that high and low WMC individuals do not necessarily differ in the overall amount of attention control that can be applied, but rather differences arise in the consistency with which control is applied. That is, low WMC individuals are more likely to demonstrate lapses or fluctuations in attention than high WMC individuals. This view suggests that mean differences in performance are largely due to the fact that low WMC individuals demonstrate more variability in their responses than high WMC individuals. Thus, on some trials high and low WMC individuals should actually perform equivalently, 
whereas low WMC individuals will perform more poorly on a larger proportion of trials than high WMC individuals. This view necessitates examining the overall distribution of responses rather than just looking at the mean. For example, if low WMC individuals are slower in implementing control on a constant basis than high WMC individuals, then when examining the entire distribution of responses for high and low WMC individuals we should see that the distribution for low WMC individuals is shifted over by a constant amount compared to high WMC individuals. If, however, differences are due to the fact that low WMC individuals demonstrate more lapses of attention than high WMC individuals, we should see that the overall distributions are similar, but that low WMC individuals have more skew at the slow tail of their distribution suggesting that they have more slow responses than high WMC individuals. This latter hypothesis is consistent with the worst performance rule, which suggests that the slowest reaction times (RTs) typically correlate higher with measures of cognitive abilities than the fastest RTs (see Coyle, 2003 for a review; see Ratcliff, Schmiedek, \& McKoon, 2008; Schmiedek et al., 2007 for diffusion model accounts of the worst performance rule). For example, Larson and Alderton (1990) found that the correlations between RTs on a choice RT task and composites of WMC and intelligence increased from the fastest to the slowest RTs. Thus, the slowest and the worst trials correlated the best with composites of WMC and intelligence. Similarly, Schmiedek, Oberauer, Wilhelm and Süß (2007) found that the slowest RTs from multiple-choice RT tasks were substantially related to both WMC and measures of fluid intelligence. Like the Larson and Alderton (1990) study, these results suggest that the slowest and worst trials are related to both WMC and cognitive abilities. Furthermore, during mind-wandering episodes participants demonstrate increased response variability and increased fidgeting, suggesting that increased behavioral variability can be considered as an online indicator of mind-wandering (Seli, Cheyne, \& Smilek, 2013; Seli et al., 2014).

By examining RT distributions on attention control tasks, more recent research has directly examined whether WMC is related to performance across the board, or differences are more isolated to the slowest responses. For example, examining the full distribution of RTs in the psychomotor vigilance task (a sustained attention task), Unsworth et al. (2010) found that WMC was related to the slowest but not the fastest RTs. In subsequent work we have found that the slowest $20 \%$ of trials on this task correlates well with typical measures of attention control such as performance on the antisaccade, Stroop, SART, and flanker tasks (Unsworth \& Spillers, 2010; Unsworth \& McMillan, 2014). This suggests that on most of the trials, high and low WMC individuals perform the same, but that low WMC individuals have disproportionally more slow responses than high WMC individuals. As noted above, recent work examining subjective reports of task-unrelated thoughts (such as reports of mind-wandering or external distraction) corroborate this idea by demonstrating that low WMC individuals report more instances of task-unrelated thoughts than high WMC individuals both in the laboratory and in real-world situations (McVay \& Kane, 2012a; Unsworth, Brewer, \& Spillers, 2012). Importantly, when high and low WMC individuals report being on task (i.e., they are not mind-wandering or distracted) performance tends to be equivalent. Like the results from examining RT distributions, these results suggest that low WMC individuals can perform just as well as high WMC individuals most of the time. However, low WMC individuals tend to be more inconsistent in their performance than high WMC individuals due to fluctuations and lapses in attention control as observed in both laboratory settings and daily life. For example, shown in Fig. 2 are RTs for one typical high WMC and one typical low WMC individual on the psychomotor vigilance task (Fig. 2a) and the Stroop task (Fig. 2b). As can be seen, most of the time the low WMC individual has just as fast RTs as the high WMC individual. However, the low WMC individual experiences more frequent lapses of attention (perhaps due to mind-wandering), leading to occasional very slow RTs.

In a recent study McVay and Kane (2012b) directly examined the notion that lapses of attention in the form of mindwandering resulted in particularly slow RTs and mediated the relation with WMC. McVay and Kane found that the slowest RTs are negatively correlated with WMC, but positively correlated with mind-wandering rates. Importantly, they found that mind-wandering rates partially mediated the relation between WMC and the slowest responses. Note that on some tasks lapses of attention can actually lead to very fast RTs as well as slow RTs. Specifically, on go/no-go tasks that promote premature responding, it is possible that both the fastest and slowest RTs are indicative of lapses of attention (Cheyne et al., 2009; McVay \& Kane, 2012b; Unsworth, 2015). Furthermore, relying on evidence-accumulator models (specifically the linear ballistic accumulator model; Brown \& Heathcote, 2008), McVay and Kane (2012b) found that trial-to-trial variability in drift rate correlated with WMC and mindwandering rates. Thus, low WMC individuals demonstrated more inconsistent performance than high WMC individuals, and part of the reason for this inconsistency was due to mind-wandering.

Building on these ideas, Unsworth (2015) re-examined prior studies demonstrating a relation between WMC and attention control, by examining whether consistency of attention control was a key factor. Unsworth found that variability of RTs in attention control tasks, but not variability in RTs on lexical decision tasks, correlated with WMC. Furthermore, variability in RTs on attention control tasks predicted everyday attention failures and was related to subjective reports of mind-wandering during the attention control tasks. For example, shown in Fig. 3 is a reanalysis of Unsworth and McMillan (2014a) 
(a)

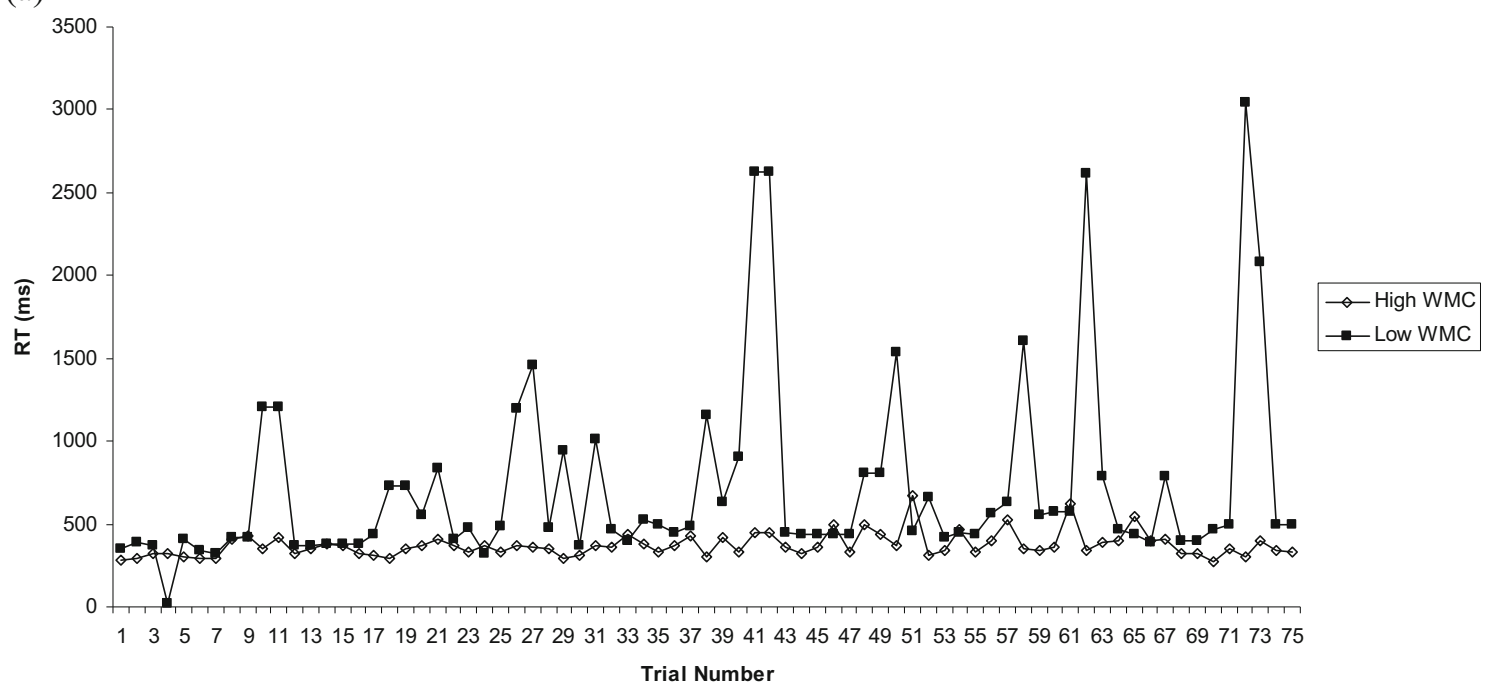

(b)

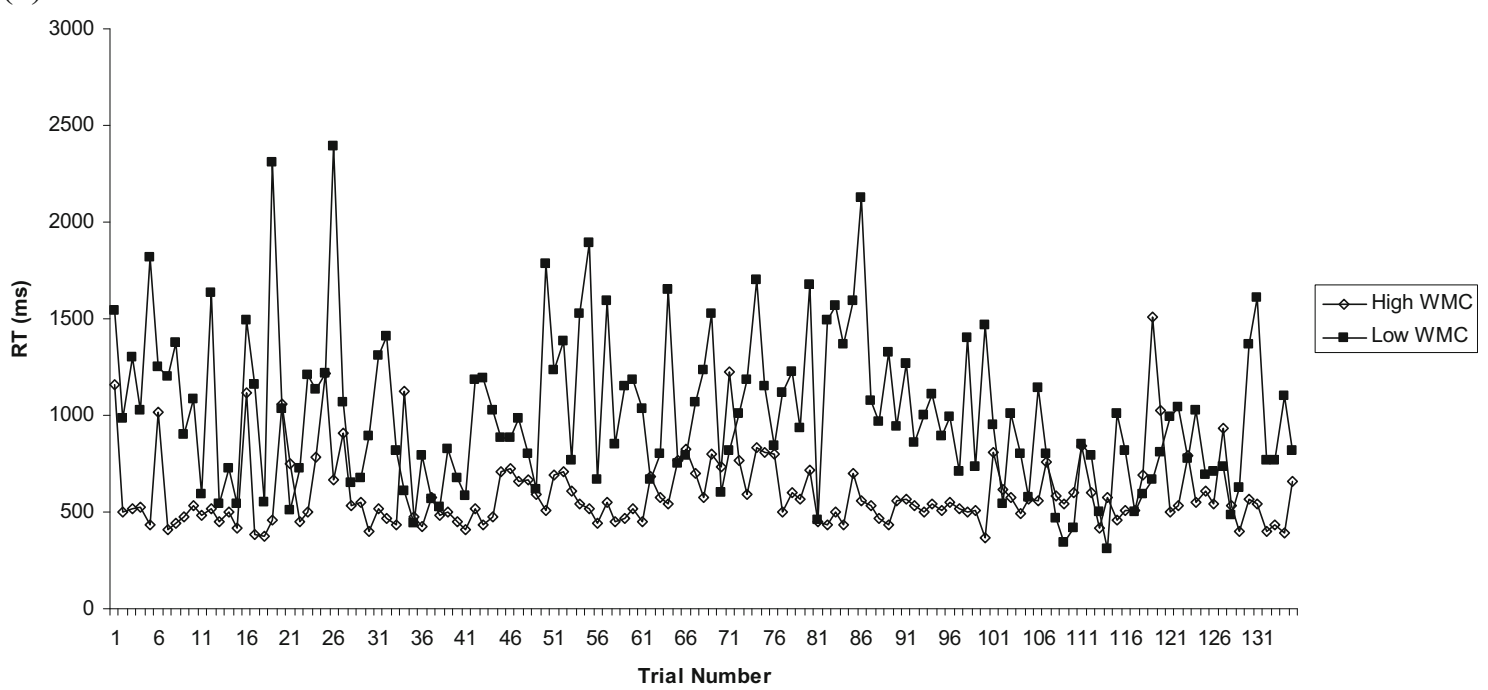

Fig. 2 (a) Trial-by-trial reaction time (RT) performance for a typical high and typical low working-memory capacity (WMC) individual on the psychomotor vigilance task. (b) Trial-by-trial RT performance for the same high and low WMC individuals on the Stroop task

demonstrating it is possible to extract a higher order attention control factor based on the shared variance from mind-wandering, performance on the attention control tasks, and variability in RTs on the attention control tasks, and this higher-order attention control factor is strongly related to WMC. Thus, like McVay and Kane (2012b), variability in RTs (particularly slow RTs) was related to WMC and mind-wandering rates (see also Kane et al., 2016). Collectively this suggests that lapses or fluctuations of attention are one of the critical determinants of the relation between WMC and attention control.

Although we have primarily focused on variability in RTs, it is important to note that there are other important indicators of lapses of attention and these different indicators might be related to different states of task disengagement. For example, Cheyne et al. (2009) suggested that variability in RTs indexed a state of relative inattention during the SART, whereas anticipatory RTs (very fast go RTs) were associated with a deeper level of zoning out during the SART, and finally omission errors on go trials represented an overall deeper level of task disengagement. In support of this, Cheyne et al. (2009) found that these three indicators of lapses were all strongly correlated, but importantly each accounted for unique variance in no-go errors, suggesting that the states were somewhat distinct, although a reanalysis of their data suggest that the majority of the variance in no-go errors was actually shared by the three indicators. Regardless, it is clear that there are various behavioral (as well as physiological) indicators of lapses of attention. Future research is needed to examine the extent to which these various indicators reflect the same overall construct or different states of attentional disengagement, and their relation with individual differences in WMC and attention control more broadly. 


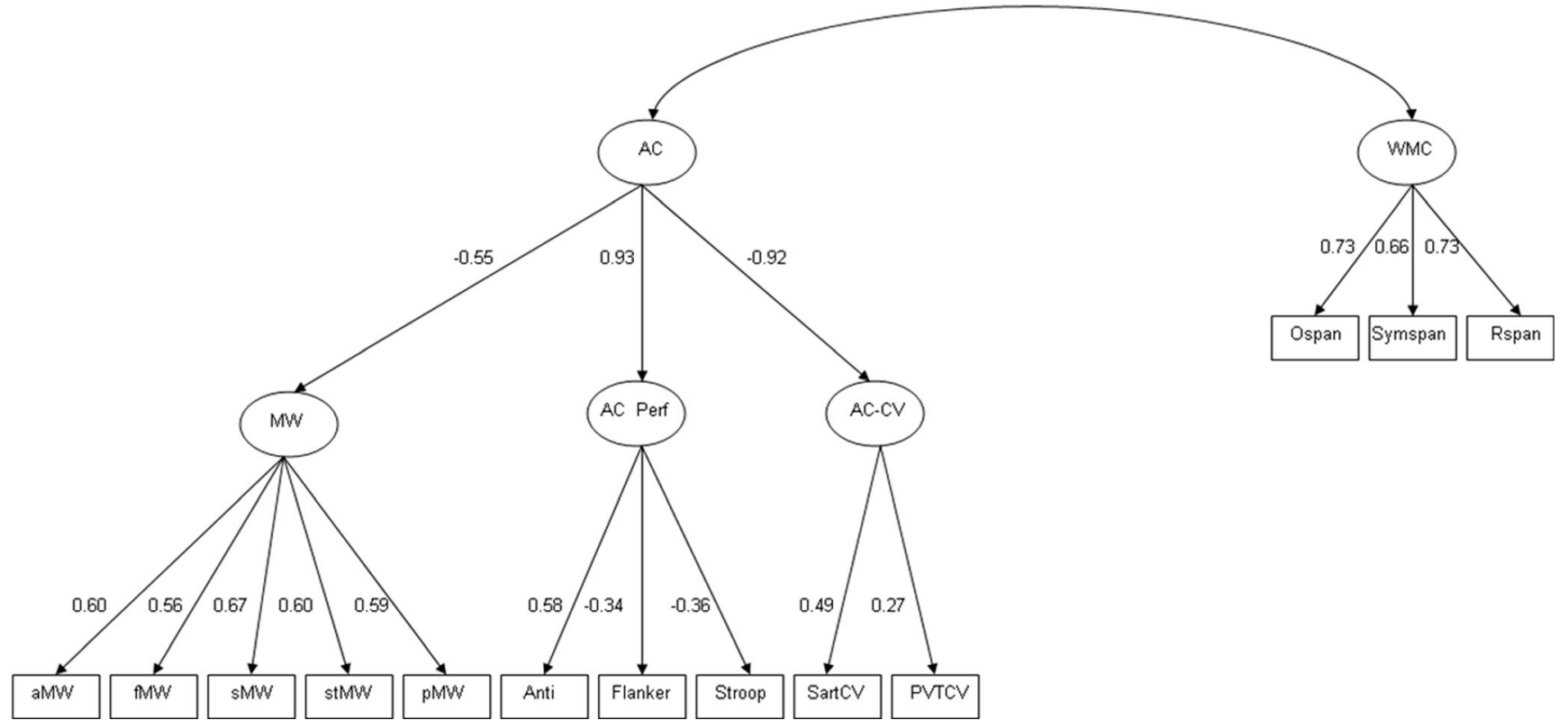

Fig. 3 Confirmatory factor analysis examining relation between working memory capacity (WMC) and a higher-order attention control (AC) factor composed of lower-order factors of self-reports of mindwandering (MW), performance on laboratory measures of attention control (AC Perf), and coefficient of variation of reaction times on laboratory measures of attention control (AC-CV). All paths are significant at the $p<.05$ level. The fit of the model was acceptable, $\chi^{2}$ $(60)=141.83, p<.001, \mathrm{RMSEA}=.08, \mathrm{NNFI}=.90, \mathrm{CFI}=.91, \mathrm{SRMR}=$ .06. Ospan= operation span recall, $R$ span= reading span recall, Symspan=

\section{Large-scale brain networks involved in working memory and attention control}

A great deal of neuroimaging work suggests the existence of three important intrinsically connected brain networks whose interactions are thought to be important for working memory, attention control, and a host of other higher-order cognitive processes (e.g., Bressler \& Menon, 2010; Menon, 2011). Specifically, these networks include the fronto-parietal control network (or central executive network), the default mode network, and the salience network. Here we briefly review each of these networks and highlight their roles in working memory, attention control, and individual differences therein. Furthermore, we acknowledge that these are complex networks with multiple complex interactions and that our review will be somewhat simplified. It is beyond the scope of the current paper to detail all aspects of these putative networks.

As the name suggests, the fronto-parietal control network (FPN) consists of frontal and parietal regions that are critically important for working memory and attention control (Cohen et al., 2004; Corbetta \& Shulman, 2002; Dosenbach et al., 2008; Fox et al., 2005; Miller \& Cohen, 2001; Peterson \& Posner, 2012; Vincent et al., 2008). In particular, the FPN is involved in a wide array of cognitively demanding tasks that require the active maintenance of task goals, particularly in the symmetry span recall, Anti $=$ antisaccade accuracy, Flanker $=$ Flanker interference score, Stroop $=$ color word Stroop interference score, SartCV $=$ sustained attention to response task coefficient of variation for correct reaction times, $P V T C V=$ psychomotor vigilance task coefficient of variation for correct reaction times, $a M W=$ mind-wandering rates Antisaccade, $f M W=$ mind-wandering rates Flankers, $s M W=$ mindwandering rates Stroop, $s t M W=$ mind-wandering rates SART, $p M W=$ mind-wandering rates psychomotor vigilance task

presence of interference and distraction (Niendam et al., 2012). Initial work on the executive attention theory of WMC suggested that individual differences in WMC were primarily due to differences in the prefrontal cortex (Kane \& Engle, 2002). Subsequent work has largely corroborated these ideas, but has also demonstrated that the greater FPN seems to be important. For example, prior research has shown that the FPN is extensively activated during complex working memory span tasks compared to baseline tasks (Bunge et al., 2000; Chein et al., 2011; Faraco et al., 2011; Kondo et al., 2004; Osaka et al., 2003; Smith et al., 2001). Importantly, activity in FPN tends to correlate with performance on the complex span tasks, such that greater FPN activations are linked with better performance suggesting that higher WMC individuals tend to better utilize the FPN during the tasks (Faraco et al., 2011; Osaka et al., 2003). Similar activation patterns as well as EEG signals and individual differences are also found on visual change detection tasks, which are another potent measure of individual differences in WMC (McNab \& Klingberg, 2008; Minamoto, Yaoi, Osaka, \& Osaka, 2015; Todd \& Marois, 2004, 2005; Vogel \& Machizawa, 2004; Vogel, McCollough, \& Machizawa, 2005). Additionally, individual differences in FPN activity have been shown to be an important predictor of individual differences in behavioral measures of cognitive ability including independent measures of WMC, 
attention control, and even fluid intelligence (Burgess, Conway, Gray, \& Braver, 2011; Gray, Chabris, \& Braver, 2003; Unsworth et al., 2015). Finally, FPN is thought to be critically important for overall intelligence levels as specified in the parieto-frontal integration theory of intelligence (Colom et al., 2009; Jung \& Haier, 2007). Thus, it is clear that the FPN is critically important for working memory and attention control likely by actively maintaining important task-relevant information in a highly active state in the presence of interference and distraction. Furthermore, individual differences in FPN seem to be linked with individual differences in WMC, attention control, and intelligence.

Although much prior work has focused on the role of FPN in working memory and attention control, recent research suggests that the FPN is just one of several interacting networks that are critical for task engagement. In addition to the FPN, the default mode network (DMN) has recently been shown to be important for aspects of attentional and cognitive control. The DMN consists of a broad array of areas (middle prefrontal cortex, lateral frontal cortex, medial parietal cortex, medial temporal lobe, and lateral temporal cortex) that tend to be active during rest periods and/or when one is engaging in internally driven cognitive processes such self-generated thoughts, mind-wandering, autobiographical memory retrieval, etc. (Andrews-Hanna, Smallwood, \& Spreng, 2014; Raichle et al., 2001). In most attention control tasks where attention is directed externally, the FPN shows increases in activation with task demands, the DMN shows decreases in activation. Thus, in demanding external attention tasks, the FPN suppresses DMN (negative correlation). However, for internally driven tasks such as planning, imagery, or autobiographical memory retrieval, the FPN and DMN are positively correlated. Likewise, when external tasks require retrieval or access of information from memory the FPN and DMN tend to act together (Konishi et al., 2015; Smallwood et al., 2013; Spreng et al., 2014; Vatansever et al., 2015). Thus, it is not always the case that the FPN suppresses the DMN. However, as noted by Spreng et al. (2014), in most attention control or cognitive control tasks "engagement of the DMN is incongruent with tasks goals" (p. 14108). Thus, the FPN is needed to suppress the DMN to prevent potentially distracting thoughts from interfering with task performance. Important for the current discussion is the finding that competition between FPN and DMN is linked to lapses of attention, inconsistency in attention control, and subjective reports of mind-wandering. For example, Weissman et al. (2006; see also Chee et al., 2008) found that the slowest responses in an attention-demanding task were associated with lower activation in FPN. Weissman et al. suggested that this reduced activity reflected a lapse of attention whereby participants were focusing on internal thoughts (mind-wandering) rather than the task at hand prior to the onset of the trial. Weissman et al. (2006) further found that the slowest responses were associated with increased DMN activity. ${ }^{1}$ Weissman et al. (2006) argued that this increased activity reflected taskirrelevant thoughts (such as daydreaming), which led to a lapse of the task goal and a subsequent decrement in goal directed behavior. Indeed, Mason et al. (2007) found that greater self-reports of mind-wandering were related to greater DMN activity and DMN activity was positively correlated with a daydream frequency scale. Thus, DMN activity was related to not only the slowest responses (indicative of lapses of attention), but also to self-reports of mind-wandering with those individuals who daydream the most having the greatest DMN activity.

Similar results have been demonstrated by Drummond et al. (2005), who found that the slowest responses in a sustained attention task (the psychomotor vigilance task described previously) were positively related with DMN activity, and suggested that this increased activity in the DMN network reflected instances of task disengagement and lapses of attention. Likewise, Kelly, Uddin, Biswal, Castellanos, and Milham (2008) and Esterman et al. (2012) have found that greater DMN activity is associated with greater variability in response times, suggesting that more inconsistency in attention control is associated with greater DMN activity. In fact, Kelly et al. found that the greater the negative correlation between FPN and DMN, the more consistent behavior was. The weaker the negative correlation between FPN and DMN, the more inconsistent behavior became. Thus, in those situations in which the FPN adequately suppressed the DMN, behavioral performance was consistent and there were few lapses of attention. However, in those situations in which the FPN could not adequately suppress the DMN, behavioral performance was more inconsistent and there were more lapses of attention. Recent meta-analyses have also suggested DMN activity during self-reports of mind-wandering (Fox et al., 2015). Additionally, FPN areas and salience network areas (see below) have also routinely been found to be active during mind-wandering. These networks may be needed to maintain mind-wandering (FPN and DMN coupling) or they may be needed to detect mind-wandering and lapses of attention and to reorient attention back to the current task.

Collectively these results suggest that during attention control tasks lapses of attention and mind-wandering are related to reduced activity in FPN and increased activity in DMN, which

\footnotetext{
${ }^{1}$ Note that we are not suggesting that the slowest responses will always be linked with greater DMN activity. Rather on attention-control tasks where attention must be focused on external information and distracting information kept at bay, the FPN will need to suppress the DMN to prevent lapses of attention. In those situations where the FPN and DMN act in concert, greater activity in the DMN will be linked with faster (not slower) RTs (Smallwood et al., 2013).
} 
lead to decrements in goal-directed behavior. Thus, in many laboratory attention control tasks where attention has to be allocated to external stimuli (typically presented on a computer screen) and perceptual decoupling will result in reduced task performance, interactions between the FPN and DMN are critically important for success. The FPN is needed to maintain task goals and prevent lapses of attention (such as mind-wandering) by suppressing the DMN in order to fully allocate attention to the external environment. Furthermore, recent research into individual differences has suggested that individuals with higher WMC demonstrate stronger anticorrelations between FPN and DMN (Keller et al. 2015). Thus, although the DMN is important for many internal tasks, DMN activity can be problematic when attention needs to be fully allocated to external tasks and any lapse of attention can harm performance. That is, lapses of attention likely "occur in moments when the mind configures mental resources so as to perform an internal task that is incompatible with current behavioral goals" (p. 125; Smallwood et al., 2013).

In addition to the roles of the FPN and DMN in attention control and working memory, the salience network also seems critically important. The salience network ( $\mathrm{SN}$ ) includes the dorsal anterior cingulate cortex and the frontal operculum/ anterior insula cortex, and is thought to be important for identifying motivationally salient and important stimuli (Menon \& Uddin, 2010; Seeley et al., 2007; similar to the ventral attention network proposed by Corbetta \& Shulman, 2002). In most task-based functional imaging studies (fMRI) the SN and FPN are coactive, demonstrating increasing activation levels, while the DMN demonstrates decreasing activation. Theoretically the SN is important for mediating dynamic interactions between other brain networks such as the FPN and DMN and switching between these networks (Menon \& Uddin, 2010; Seeley et al., 2007; Sridharan, Levitin, \& Menon, 2008). In particular, it is thought that the SN detects salient stimuli (either externally or internally based) and marks those stimuli for further processing and initiating control signals to bias processing of relevant stimuli and ignore irrelevant stimuli. That is, the SN produces a transient signal that engages working memory and attention control processes (i.e., FPN) while disengaging systems that are not task-relevant (i.e., DMN; Sridharan, Levitin, \& Menon, 2008). For example, Bonnelle et al. (2012) found that deficits in DMN deactivations were predicted by the amount of white matter damage in the SN. Thus, the SN is critically important for regulating the DMN and deficits in regulating the DMN can lead to poor attention control and poorer task performance. The $\mathrm{SN}$ is, therefore, critical in determining which events are salient and require additional allocations of attention, thereby furthering processing of those events leading to desirable behaviors. Furthermore, recent research suggests that performance on attention control tasks is correlated with SN activity, suggesting that individual differences in attention control are partially due to differences in SN activity (Cai et al., 2016). Recent theorizing suggests that errors (and potentially lapses of attention) are a particularly salient event that the SN responds to, leading to adjustments in control by other networks (Ullsperger et al., 2010). Thus, consistent with research on conflict monitoring (Botvinick et al., 2001), the SN may be particularly important in terms of detecting errors and lapses of performance and signaling control areas to mobilize resources to bring performance back on track.

\section{The locus coeruleus norepinephrine system and regulating attentional state}

Recent research suggests that the locus coeruleus norepinephrine system (LC-NE) may be particularly important in modulating FPN representations based on attentional control demands (Cohen, Aston-Jones, \& Gilzenrat, 2004). The LC is a brainstem neuromodulatory nucleus that is responsible for most of the NE released in the brain, and it has widespread projections throughout the neocortex including areas associated with the FPN and SN (Berridge \& Waterhouse, 2003; Samuels \& Szabadi, 2008a; Szabadi, 2013). The LC also receives major inputs from the prefrontal cortex (particularly the anterior cingulate cortex) suggesting a reciprocal connection between the LC-NE system and the FPN and SN (Arnsten \& Goldan-Rakic 1984; Jodo et al., 1998; Rajkowski, Lu, Zhu, Cohen, \& Aston-Jones, 2000). Given these wide projections throughout neocortex, the LC-NE system is critically important for modulating neural processing in different networks. It is thought that LC-NE activity enhances signal-to-noise ratios in target neurons by decreasing spontaneous firing and by potentially increasing firing for salient events (Aston-Jones \& Cohen, 2005; Berridge \& Waterhouse, 2003; Foote et al., 1980). In neurocomputational models this increases the gain of these neurons, which allows the system to selectively respond to relevant inputs while ignoring irrelevant inputs (Aston-Jones \& Cohen, 2005; Servan-Schreiber, Printz, \& Cohen, 1990; Usher et al., 1999). Under high gain states excited neurons become more active and inhibited neurons become less active. Conversely, under low gain states there is less activation for excited neurons and more inhibited neurons are not effectively suppressed, leading to more neural noise. Thus, the LC-NE system acts as a modulatory system to the FPN (and other networks) in which resources are mobilized to ensure greater processing of target information while ignoring irrelevant or distracting information and reducing neural noise.

Generally the LC-NE system has been associated with general functions such as the sleep-wake cycle and overall arousal levels (Berridge \& Waterhouse, 2003; Samuels \& Szabadi, 2008b; Szabadi, 2013). In particular, the LC-NE system is 
important for determining general arousal state and attentional interest. Within the LC-NE system neurons demonstrate two modes of firing: tonic and phasic. Tonic activity refers to the overall baseline activity and phasic activity refers to the brief increase in firing rate associated with salient stimuli. A great deal of recent research suggests that there is an inverted-U relationship between LC tonic activity and performance on various cognitive tasks consistent with the Yerkes-Dodson curve (Yerkes \& Dodson, 1908). Specifically, it is assumed that when tonic LC activity is low (hypoarousal), individuals are inattentive, non-alert, and disengaged from the current task leading to poor behavioral performance and little to no phasic LC activity in response to task-relevant stimuli. As tonic LC activity increases to an intermediate range (phasic mode), attention becomes more focused, LC phasic activity increases for target stimuli, and behavioral performance is optimal. The LC-NE system seems particularly sensitive to salient taskrelated stimuli that results in increased phasic activity to those stimuli when tonic activity is at intermediate levels (AstonJones \& Cohen, 2005; Berridge \& Waterhouse, 2003). However, as tonic LC activity increases further, the individual experiences a more distractible attentional state (hyperarousal and stress), leading to task disengagement, lowered LC phasic activity, and a reduction in behavioral performance. Intracranial recordings in rats and monkeys and psychopharmacological studies in animals and humans provides evidence in support of the notion of an inverted-U relationship between the LC-NE system and behavioral performance (Aston-Jones \& Cohen, 2005; Berridge \& Waterhouse, 2003; Chamberlain \& Robbins, 2013; Ramos \& Arnsten, 2007). Thus, the increase in signal-to-noise ratio (gain) is achieved via interactive tonic and phasic activity. Too much or too little tonic activity leads to little phasic firing (more neural noise) and poor attention control, whereas optimal levels of arousal and attention are achieved via intermediate tonic activity and maximal phasic activity (greater signal and less noise). Thus, the LC-NE is critically important for regulating attentional state in the FPN via synergistic tonic and phasic activity.

The importance of the LC-NE system to working memory and attention control is further bolstered by psychopharmacological studies. For example, a number of studies have shown that drugs such as modafinil increase central NE leading to increases in subjective alertness and performance on some attention control and working memory measures (see Chamberlain \& Robbins, 2013 for a review). Modafinil has also been shown to be related to deactivation of DMN during task performance (Minzenberg, Yoon, \& Carter, 2011). Drugs such as clonidine decrease central NE leading to lowered subjective alertness and decreased performance on measures of working memory and attention control (see Chamberlain \& Robbins, 2013, for a review). Furthermore, these pharmacological manipulations typically depend on baseline levels of arousal, suggesting the importance of tonic NE levels in determining attentional state (Coull et al., 2004; Smith \& Nutt, 1996). Finally, dysregulation of the LC-NE system has been implicated in a number of disorders including Alzheimer's disease, Parkinson's disease, attention deficit hyperactivity disorder, autism, schizophrenia, and post-traumatic stress disorder, among others (e.g., Arnsten, 2009; Aston-Jones et al., 2007; Berridge \& Waterhouse, 2003; Frank et al., 2007; Imeraj et al., 2012; Killeen, Russell, \& Sergeant, 2013; Ressler \& Nemeroff, 2001; Robertson, 2013; Vazey \& AstonJones, 2012) as well as cognitive decline associated with normal aging (Mather \& Harley, 2016).

Collectively this research demonstrates the importance of the LC-NE system in modulating FPN activity and regulating attention control and working memory processes. Furthermore, this work suggests that the LC exhibits fluctuations between various states during basic attentional tasks, and these fluctuations are linked to fluctuations in behavioral performance. As such, prior research suggests the LC-NE system is important in regulating the current attentional state and that fluctuations in attentional state may be partially due to fluctuations in tonic and phasic LC activity.

\section{A LC-NE account of WMC and attention control}

Above we suggested that individual differences in WMC and attention control are intimately related both in and out of the laboratory. Furthermore, we suggested that much of this relation seems to be due to fluctuations in attention control such that low WMC individuals experience more fluctuations in attention control than high WMC individuals, leading to periodic episodes of goalneglect and more variable and erratic performance. In particular, we suggested that these fluctuations or lapses of attention control arise when participants are disengaged from the primary task, resulting in mindwandering or external distraction. That is, in those situations where attention is not tightly focused on the current task goal, internal (mind-wandering) or external distractors can capture attention, resulting in poorer behavioral performance. Here we propose a LC-NE account of individual differences in WMC and attention control suggesting that differences in LC-NE functioning constitute a major reason for individual differences in these two constructs. Given the importance of the LC-NE system for regulating attentional state and temporal variations in attention control, it seems highly likely that variation in LC-NE functioning is associated with individual differences in WMC and attention control. Below we 
examine the potential role of other important neurotransmitters (such as dopamine and acetylcholine), but our focus is primarily on the LC-NE system. Specifically, we suggest that attention control failures are due to a dysregulation of LC-NE functioning in which there is an inability to maintain moment-to-moment optimal tonic levels of NE resulting in a dysregulation of phasic activity. These fluctuations in tonic LC activity lead to moment-to-moment fluctuations in the ability of the FPN to control attention in a goal-directed manner. As shown in Fig. 4, when LC-NE tonic levels are optimal the LC modulates the FPN so that the FPN is fully engaged and attention is fully allocated to goal-directed processing. With externally driven attention control tasks this results in a suppression of the DMN (suppressing mind-wandering), the active maintenance of the task goal in working memory, a strong phasic LC response, and fast and accurate performance. Thus, the LC determines moment-to-moment task engagement levels by modulating the FPN, which can effectively suppress the DMN (in some situations), resulting in correct performance. However, when LC-NE tonic levels are too low (under aroused/inattentive) or too high (anxious/stressed) the LC does not adequately modulate the FPN, leading to weakened FPN activity and a decreased allocation of attention to processing. In this case the DMN is not fully suppressed and internally generated thoughts (mindwandering) are more likely to interfere, leading to goalneglect, weakened phasic LC responding, and slower and more error-prone performance. Thus, lapses of attention are due to a cascade of events in which the LC does not fully modulate the FPN, which in turn does not fully

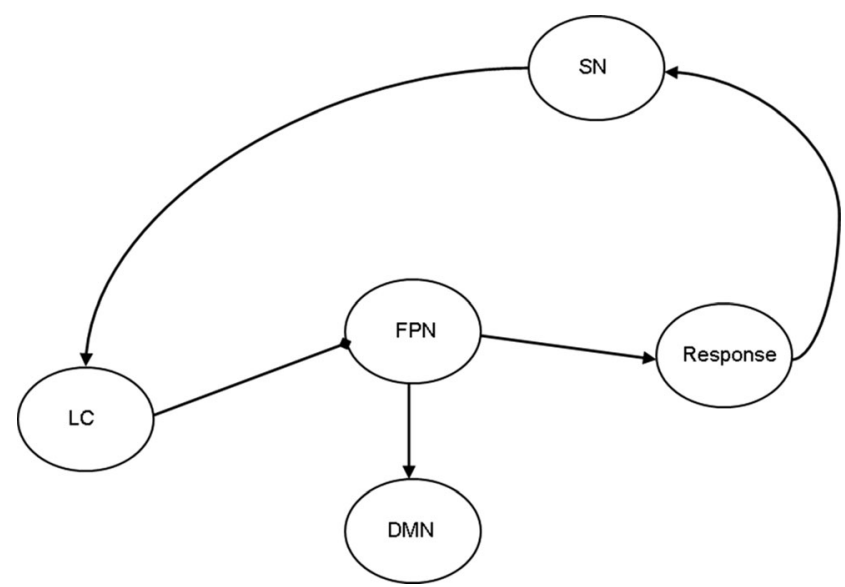

Fig. 4 Model in which the locus coeruleus (LC) modulates the frontoparietal network (FPN), which in turn suppresses the default-mode network (DMN) during tasks that require focused external attention. The salience network ( $\mathrm{SN}$ ) detects lapses in performance (errors and lapses of attention) and sends feedback to the LC resetting overall arousal levels and getting the LC back online suppress the DMN, leading to increased instances of mind-wandering. ${ }^{2}$ In such cases if the behavioral response is an error or an exceptionally long RT (or if the participant is aware they are mind-wandering), the $\mathrm{SN}$ is activated resulting in an orienting-like response in which the SN sends feedback to the LC suggesting a change in arousal, an increase in the allocation of attentional resources, and subsequent increase in behavioral performance on the next trial (Cohen et al., 2004; Ridderinkhof \& Harsay 2012; Ullsperger et al., 2010). Thus, following particularly salient events (such as errors or lapses of attention), the LC (based on information from the $\mathrm{SN}$ ) reorients or resets the overall arousal levels, getting the LC back online (Corbetta et al., 2008; Sara \& Bouret, 2012).

This model is based on earlier attention and cognitive control models by Kahneman (1973) and Aston-Jones, Cohen and colleagues (Aston-Jones \& Cohen, 2005; Cohen et al., 2004) as well as recent work examining large-scale brain networks and their role in attention control (Corbetta \& Shulman, 2002; Dosenbach et al., 2006; Menon \& Uddin, 2010; Miller \& Cohen, 2001; Petersen \& Posner 2012). In particular, like the Kahneman (1973) and Aston-Jones and Cohen (2005) models, the current model suggests the important role of arousal on attention control and the importance of performance monitoring for detecting lapses in performance and providing feedback to arousal structures to change attention allocation policies based on current performance (see also Hockey, 1997).

In terms of individual differences we suggest that low $\mathrm{WMC}$ and low attention control individuals demonstrate fluctuations in attention due to a dysregulation of the LC-NE system compared to high WMC and high attention control individuals. We suggest that low WMC individuals have more moment-to-moment task disengagement than high WMC individuals resulting in more erratic performance, less

\footnotetext{
${ }^{2}$ As noted previously, mind-wandering and lapses in these situations is likely due to spontaneous (unintentional) mind-wandering, rather than deliberate (intentional) mind-wandering where resources are deliberately directed internally (Grodsky \& Giambra, 1990-1991; Seli et al., 2016). The LC-NE system is likely important for both types of mind-wandering (Lenartowicz, Simpson, \& Cohen, 2013; Mittner et al., 2016). In particular, spontaneous mindwandering is likely associated with lowered LC tonic activity associated with inattentiveness and lowered levels of alertness and arousal (Lenartowicz, Simpson, \& Cohen, 2013). Spontaneous mind-wandering may also be related to elevated tonic LC activity associated with a more exploratory state (Mittner et al., 2016). Deliberate mind-wandering, however, is more likely associated with intermediate LC activity, indicating an optimal state of arousal where attention is deliberately allocated internally, rather than to the external task at hand resulting in active mind-wandering (Lenartowicz, Simpson, \& Cohen, 2013; Mittner et al., 2016). As discussed below, it should be possible to detect these various types of mind-wandering using pupillometry. Additionally, deliberate mind-wandering is more likely associated with communication and cooperation between the FPN and DMN than spontaneous mind-wandering, suggesting that attention control resources are likely being directed internally during deliberate mind-wandering episodes (Golchert et al., in press).
} 
sensitivity to task-relevant stimuli, and more sensitivity to task-irrelevant stimuli (both internal and external). Given dysregulation of LC-NE functioning, the FPN for low WMC individuals is only weakly activated resulting in increased DMN activity and increased mind-wandering than high WMC individuals. Note, we are not just saying more DMN activity overall for low WMC individuals compared to high WMC individuals. Rather LC dysregulation causes a weakened FPN response more readily for low WMC individuals than for high WMC individuals. When the FPN needs to direct attention to external information, this greater DMN interference results in more mind-wandering and poorer behavioral performance for low WMC individuals than for high WMC individuals. However, when the FPN needs to direct attention internally (such as during planning, imagery, autobiographical memory retrieval, etc.) and thus greater coupling between FPN and DMN, greater LC dysregulation would result in a weakly activated DMN. Thus, in some situations we would expect low WMC individuals to demonstrate more DMN activity, whereas in other situations we would expect high WMC individuals to demonstrate more DMN activity. Because the FPN is weakly activated and the DMN is weakly suppressed (resulting in more mind-wandering), low WMC individuals should demonstrate more errors on tasks that require effortful attention control, more variability in responding on such tasks, and higher rates of subjective mind-wandering. As reviewed previously, this is exactly what has been found across a number of prior studies. The current theory suggests that differences in WMC and attention control may be due to differences in LC-NE functioning which in turn result in differences in FPN functioning. Thus, differences in LC-NE drive differences in FPN and interactions with DMN.

Within this framework there are several possibilities for why low WMC individuals demonstrate a dysregulation in LC-NE functioning. As shown in Fig. 5a, and consistent with the notion that there is an inverted U-function relating LC-NE functioning and performance, it is possible that low WMC individuals have overall lower tonic LC-NE (and lower phasic) firing compared to high WMC individuals. This would suggest that low WMC individuals are constantly in a hypoactive mode of LC tonic activity associated with inattentiveness and lowered levels of alertness and arousal. Overall this would result in the LC weakly modulating the FPN resulting in lowered attention control. If attention is focused externally and there are potent external distractors (such as flashing lights in the antisaccade task or hearing your name in a dichotic listening task), this would result in external distraction and attentional capture via bottom-up sources (Lenartowicz, Simpson, \& Cohen, 2013). If attention is weakly focused on the external task and there are strong internal concerns, then attention will be captured internally, resulting in mind-wandering (Lenartowicz et al., 2013; McVay \& Kane, 2010). In both cases low WMC individuals would

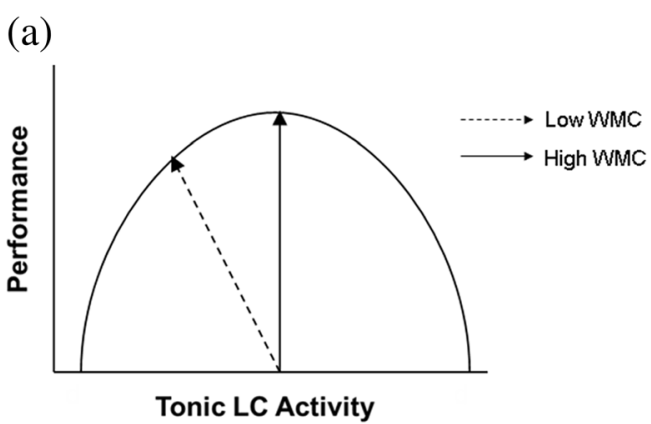

(b)

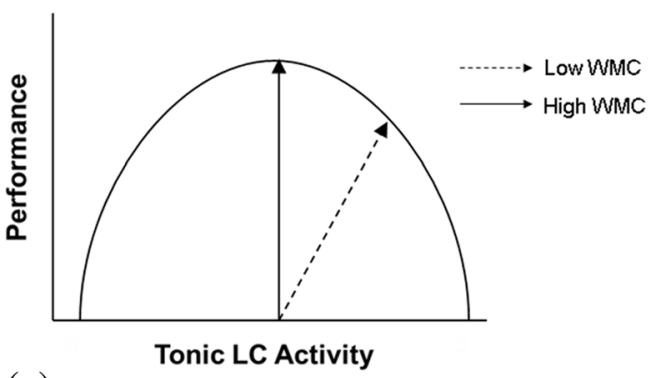

(c)

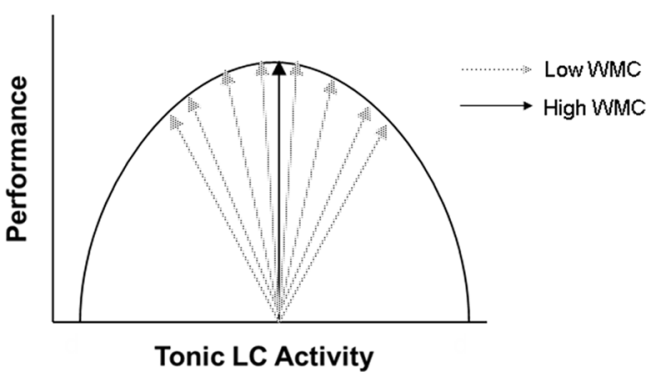

Fig. 5 Three possibilities linking low working memory capacity (WMC) to deficits in attention control. (a) Low WMC individuals have lower tonic locus coeruleus (LC) activity levels than high WMC individuals. (b) Low WMC individuals have higher tonic LC activity levels than high WMC individuals. (c) Low WMC individuals have more variable tonic LC activity levels than high WMC individuals

demonstrate poorer performance on a variety of attention control tasks compared to high WMC individuals. Thus, LC dysregulation in the form of too little tonic LC activity can lead to a weakly activated FPN and reduced attention control.

As shown in Fig. 5b, another possibility for LC-NE deficits in low WMC individuals is because rather than having too little tonic LC activity, they may have too high tonic LC activity (and too low phasic activity). This would suggest that low WMC individuals are constantly in a hyperactive mode of tonic LC activity associated with task disengagement, anxiety, stress, and impulsivity linked to increased arousal levels (Aston-Jones \& Cohen, 2005; Lenartowicz et al., 2013; Ramos \& Arnsten, 2007). This would result in the LC modulating the FPN too strongly and thus an overactive FPN resulting in disrupted attention control. If attention is focused externally this would result in an inability to discriminate target and distractors (Lenartowicz, Simpson, \& Cohen, 2013). If attention is focused internally this could result in rumination 
and racing thoughts related to stress and anxiety (Lenartowicz et al., 2013). In this hyperactive mode this would result in less task engagement for low WMC individuals, which would then lead to poorer performance on a variety of attention control tasks compared to high WMC individuals. Thus, LC dysregulation in the form of too much tonic LC activity can lead to an overactive FPN, task disengagement, and disrupted attention control.

Another possibility is that rather than having too high or too little tonic LC activity, low WMC individuals fluctuate between different LC modes more readily than high WMC individuals. That is, as shown in Fig. 5c, low WMC individuals fluctuate between optimal, too little, and too much activity throughout a task more than high WMC individuals. This would mean that much of the time low WMC individuals would perform just as well as high WMC individuals given that they would typically have similar tonic LC activity. However, given moment-to-moment fluctuations in tonic LC activity, low WMC individuals would be more likely to have lapses of attention associated with either too low or too high LC activity levels compared to high WMC individuals. This would result in greater susceptibility to external and internal distraction as on some trials the FPN would be either weakly activated or too strongly activated leading to overall more task disengagement. This fluctuations hypothesis would suggest that low WMC individuals should experience more lapses of attention, more erratic and variable performance, greater susceptibility to pre-potent responses, and overall greater levels of mind-wandering. Thus, LC dysregulation in the form of more fluctuations between LC states would result in more variable FPN (and DMN) activity, periodic failures of attention control, and overall worse performance on a number of attention control tasks.

In each of these possibilities low WMC is associated with LC dysregulation and poorer attention control due to an inability of the LC to properly modulate the amount of control via the FPN. Thus, LC dysregulation is a likely reason for poorer attention control seen in many low cognitive ability participants. The current theory suggests that individual differences in LC-NE functioning give rise to individual differences in WMC and attention control. Of course, we should also acknowledge that a fourth possibility of no relation between LC-NE functioning and individual differences in WMC and attention control is also possible. This null possibility would suggest that individual differences in WMC and attention control are due to something other than functioning of the LC-NE system. Despite the many linkages between LC-NE functioning and $\mathrm{WMC}$ and attention control (and individual differences therein) more evidence for this line of reasoning is needed. However, there are a number of complications in attempting to evaluate the idea that individual differences in LC-NE functioning give rise, in part, to differences in WMC and attention control. In particular, given its location in the brainstem and its small size, neuroimaging of the LC is difficult. Although there have been a number of recent fMRI studies examining the LC (Alnaes et al., 2014; Minzenberg et al., 2008; Murphy et al., 2014; Raizada \& Poldrack, 2008), precise measures are still difficult to obtain. Additionally, individual differences studies utilizing fMRI on a large scale are very costly in terms of time and money. Thus, another noninvasive indirect measure of LC-NE functioning is needed. One potential candidate is pupil diameter.

\section{Pupil diameter and the LC-NE system}

Much prior research has shown that the pupil dilates in response to the cognitive demands of a task (Beatty, 1982). For example, Hess and Polt (1964) demonstrated that the pupils dilated as a function of problem difficulty in a mental multiplication task with higher peak dilations for the hardest problems. Similarly, Kahneman and Beatty (1966) demonstrated that pupillary dilation increased as more items were required for recall in a standard short-term memory task (see also Peavler, 1974). These effects reflect task-evoked pupillary responses (TEPRs) in which the pupil dilates relative to baseline levels due to increases in cognitive processing load. A number of studies have demonstrated similar TEPRs in a variety of tasks (see Beatty \& Lucero-Wagoner, 2000 for a review; see also Goldinger \& Papesh, 2012; Laeng et al., 2012 for recent reviews). These and other results led Kahneman (1973) and Beatty (1982) to suggest that TEPRs are a reliable and valid psychophysiological marker of cognitive and physical effort (e.g., Zénon et al. 2014).

A number of studies have demonstrated that TEPRs are sensitive to attention control demands and working memory load. For example, a number of prior studies have found that incongruent Stroop trials elicit a larger TEPR than congruent or neutral trials (e.g., Brown et al., 1999; Laeng et al., 2011; van Steenbergen et al., 2015). Likewise, incongruent trials on flanker and Simon tasks tend to elicit larger TEPRs than congruent trials (e.g., Geva et al., 2013; van Steenbergen \& Band, 2013; van Steenbergen et al., 2015). Similar results are found when comparing prosaccade and antisaccade trials such that antisaccade trials are associated with larger TEPRs than prosaccade trials (Karatekin, Bingham, \& White, 2010; Wang et al., 2015). Furthermore, Wang et al. (2015) found that during the preparatory interval, antisaccades were associated with greater pupil dilations than prosaccades and the greater the preparatory dilation the faster the saccade. Thus, conditions associated with greater attention control and greater preparatory attention control are associated with increased phasic pupillary responses (TEPRs). Pupil dilation has also been linked to visual search (Porter et al., 2007), AX-CPT performance (Chatham et al., 2009; Chiew \& Braver, 2013), multi-object tracking (Alnaes et al. 2014; Wright et al., 2013), 
the attentional blink (Zylberberg et al., 2012), and inhibition of return (Gabay et al., 2011), to name a few. Collectively this work suggests that TEPRs track changes in attention control demands in which generally larger control requirements elicit greater TEPRs. In a similar vein research examining pupillary responses during working memory tasks has found that as the amount of information in working memory increases so do TEPRs (e.g., Heitz et al., 2008; Kahneman \& Beatty, 1966; Peavler, 1974; Unsworth \& Robison, 2015).

These results are consistent with recent research which suggests that pupil dilations are indirectly related to the functioning of the LC-NE system (Aston-Jones \& Cohen, 2005; Eldar, Cohen, \& Niv, 2013; Gilzenrat et al., 2010; Joshi et al., 2016; Phillips et al., 2000; Samuels \& Szabadi, 2008; Varazzani et al., 2015). The LC is thought to have both excitatory sympathetic and inhibitory parasympathetic connections such that an increase in LC activity results in pupil dilation via increased sympathetic and decreased parasympathetic responses (Szabadi, 2013). Furthermore, Rajkowski et al. (1993) demonstrated a modest correlation between tonic pupil diameter and baseline firing rate of an LC neuron in a monkey. More recently, Varazzani et al. (2015) demonstrated a correlation between LC activity and pupil dilation in monkeys performing an effort production task. Additionally, Joshi et al. (2016) recently demonstrated a number of findings suggesting a relation between LC activity and pupil diameter in monkeys. For example, Joshi et al. found a correlation between LC spiking activity and pupil diameter during passive fixation, in which roughly $300 \mathrm{~ms}$ after LC spike activity the pupil tended to dilate. Similarly, local field potentials in the LC preceded pupil dilation. Finally, microstimulation of the LC resulted in pupil dilation roughly $400 \mathrm{~ms}$ after the microstimulation (see also Reimer et al., 2016). Collectively, these results suggest a consistent association between LC activity and pupil diameter.

According to Aston-Jones and Cohen (2005; see also; Gilzenrat et al., 2010; Rajkowski et al., 1993), pupil diameter provides an indirect index of LC activity. Specifically, when LC tonic levels are low and arousal is low, baseline pupil diameter is small and so are TEPRs. When individuals are hyperaroused and tonic LC levels are very high, overall baseline pupil diameter is relatively large and TEPRs are small. However, when LC tonic levels are optimal (phasic mode), overall baseline pupil diameter is at intermediate levels and TEPRs are at their largest. This is consistent with prior work suggesting that TERPs are associated with increases in processing load and effort suggesting that manipulations that lead to larger TEPRs require increases in sustained attention represented by the LC phasic mode (Beatty, 1982; Beatty \& Lucero-Wagoner, 2000). Collectively, this work suggests that baseline pupil diameter and TEPRs should provide an indirect index of LC-NE functioning. For example, prior research has demonstrated that drugs such as modafinil increase central
$\mathrm{NE}$, leading to increases in subjective alertness and increases in baseline pupil diameter (Hou et al., 2005). Drugs such as clonidine decrease central NE leading to lowered subjective alertness and lower baseline pupil diameter (Hou et al., 2005). Additionally, recent neuroimaging work has shown that activity in the LC is correlated with changes in pupil diameter (Alnaes et al. 2014; Murphy et al., 2014). Thus, there is a correlation between baseline pupil diameter and baseline firing rate of LC neurons, and drugs that increase or decrease central NE levels lead to increases or decreases in baseline pupil diameter. Collectively prior research suggests that baseline pupil diameter can be used as an indirect reporter variable of LC-NE functioning.

A number of recent studies have begun to demonstrate that pupil diameter provides an indirect index of LC-NE functioning and can be used to examine changes in control state. For example, Gilzenrat et al. (2010) demonstrated that trials that were preceded by large baseline pupil diameters were associated with poorer performance indicative of lapses of engagement corresponding to the increased LC tonic activity (see also Jepma \& Nieuwenhuis, 2011). Trials that were associated with better performance and task engagement were associated with smaller baseline pupil diameters corresponding to the intermediate LC tonic activity. Murphy et al. (2011) found an inverted U relationship between baseline pupil size and performance such that RT variability was greater when baseline pupil was very small or very large, but RT variability was lowest at intermediate baseline levels. This is consistent with prior research which has consistently shown that under conditions of fatigue or low levels of alertness, baseline pupil diameter is smaller and more variable than when alert (Hou et al., 2005; Morad et al., 2000). Specifically, in sustained attention tasks baseline pupil diameter and TEPRs tend to decrease with time on task demonstrating a vigilance decrement (Beatty, 1982b, Fried et al., 2014; Unsworth \& Robison, 2016c). Furthermore, pupil variability tends to increase with time on task (Unsworth \& Robison, 2016c). Overall, the decrease in pupil diameter and the increase in pupil variability are consistent with increases in pupillary unrest as time on task increases, suggesting that as time on task increases, alertness and arousal decrease and overall fluctuations in attention increase (Hopstaken et al., 2015a, 2015b; Lowenstein, Feinberg, \& Loewenfeld, 1963; McLaren et al., 1992; Morad et al., 2000; Unsworth \& Robison, 2016c; Wilhelm et al., 2001).

Changes in pre-trial baseline pupil diameter have also been implicated in the detection of lapses of attention. For example, examining errors on very small set sizes in a working memory task (thought to be due to lapses of attention), Unsworth and Robison (2015) found that errors were associated with much smaller than normal pre-trial baseline pupil diameters than correct trials. Furthermore, in an extended sustained attention task, Kristjansson et al. (2009) found that baseline pupil 
diameter was much smaller on trials preceding very slow RTs (indicative of lapses of attention) compared to trials where RT was close to the mean. Kristjansson et al. suggested that fluctuations in alertness resulted in variable RTs and that baseline pupil diameter provides an index of changes in alertness. More recently, van den Brink, Murphy, and Nieuwenhuis (2016) found that both large and small baseline pupil diameter, along with fluctuations in pupil diameter were associated with lapses of attention. Consistent with this, Murphy et al. (2014) found that trial-to-trial variability in drift rate correlated positively with baseline pupil diameter, suggesting that increased inconsistency in RTs is associated with pupil diameter (see also Unsworth \& Robison, 2016c). Recall that McVay and Kane (2012b) found that variability in drift rate was positively correlated with mind-wandering rates, suggesting that changes in baseline pupil diameter may be due to fluctuations in attention between on-task and off-task thoughts. Indeed, recent research suggests that mindwandering is related to both larger than normal (Franklin et al., 2013; Smallwood et al., 2012) and smaller than normal (Grandchamp, Braboszcz, \& Delorme, 2014; Mittner et al., 2014; Unsworth \& Robison, 2016c) baseline pupil diameters compared to on-task thoughts. Furthermore, mind-wandering is associated with much smaller TEPRs than on-task thoughts. Given the relation between pupil dilation and mind-wandering, one might expect that pupil dilation should be associated with DMN activity during mind-wandering. Evidence consistent with this notion comes from a recent study by Yellin et al. (2015). Specifically, Yellin et al. (2015) had participants fixate a small dot on screen for $8 \mathrm{~min}$. They found that pupil diameter fluctuated considerably during the task and participants reported a high degree of mind-wandering. Importantly, they found that spontaneous fluctuations in pupil diameter correlated positively with BOLD fluctuations in DMN areas. Thus, mind-wandering was associated with DMN activity and with changes in pupil diameter. Furthermore, Schneider et al. (2016) found that spontaneous pupil fluctuations during the resting state were significantly correlated with increased activity in the SN, FPN, and to some extent the DMN, suggesting that fluctuations in pupil dilation are indicative of changes in tonic alertness. Likewise, Breeden et al. (in press) found that spontaneous pupillary fluctuations were related to SN and DMN activity as well as to individual differences in inattentiveness measured via self-report. Collectively this work suggests that pupil diameter (both baseline and TEPRs) can be used as an indirect index of LC-NE functioning to examine lapses of attention and fluctuations in attention control, which in turn are related to DMN, SN, and FPN activity.

Changes in pupillary responses are also associated with the $\mathrm{SN}$ in terms of an orienting response to especially salient events during attention demanding tasks. For example, TEPRs are larger for errors than for correct trials on a number of tasks (e.g., Braem et al., 2015; Critchley et al., 2005; Wessel et al., 2011). Furthermore, pupillary dilations associated with errors are related to activity in the $\mathrm{SN}$ in humans (Critchley et al., 2005) and monkeys (Ebitz \& Platt, 2015). Consistent with the model described previously (Fig. 4), this suggests that after salient events such as errors or lapses of attention, the SN signals the LC to change overall arousal levels to better allocate attention to the task at hand leading to dynamic changes in performance. Collectively, prior research suggests that the pupils dilate in response to increases in effortful attention control, the pupils dilate to salient events which likely trigger increases in attention control, and fluctuations in pupillary responses are linked to lapses of attention and mind-wandering. Overall this suggests that pupillary responses (both pretrial baseline and TEPRs) can be used as a psychophysiological marker for changes in attentional state and an indirect index of LC-NE functioning.

\section{Pupillary correlates of fluctuations of attention, working memory capacity, and attention control}

According to the account put forth in the current paper, individual differences in WMC and attention control are partially due to dysregulation in LC-NE functioning. Thus, individual differences in pupil diameter (both pre-trial baseline and TEPRs) should be related to individual differences in WMC, attention control, and task-disengagement in the form of the off-task thoughts. Earlier we postulated three potential reasons for why low WMC individuals would demonstrate more LC dysregulation than high WMC individuals as depicted in Fig. 5. Specifically, we suggested that low WMC individuals may have lower tonic LC activity resulting in lowered phasic responses and lowered attention control (Fig. 5a). In terms of pupillary responses this would result in low WMC individuals having lower pre-trial baseline pupils and lower TEPRs than high WMC individuals. Evidence consistent with this hypothesis comes from prior work by Heitz et al. (2008; see Tsukahara, Harrison, \& Engle, 2016 for a recent replication) who examined individual differences in WMC and pupillary responses during a reading span task. Heitz et al. (2008) found that pupil dilation increased with increases in working memory load. Furthermore, Heitz et al. (2008) found that high WMC individuals had larger baseline pupils than low WMC individuals, suggesting that high WMC individuals were overall more aroused and prepared to engage in the task than low WMC individuals. Another possibility suggested in Fig. 5b is that low WMC individuals have higher tonic LC activity resulting in lowered phasic responses and lowered attention control possibly due to anxiety and stress. In terms of pupillary responses this would result in low WMC individuals having larger pre-trial baseline pupils and lower TEPRs than high WMC individuals. Another possibility suggested in Fig. $5 \mathrm{c}$ is 
that low WMC individuals have more moment-to-moment fluctuations in tonic LC activity resulting in LC tonic activity that is sometimes too low and sometimes too high. In terms of pupillary responses, this would suggest that low WMC individuals should have more variability in pupil diameter (both pre-trial baselines and TEPRs) than high WMC individuals. Evidence consistent with this hypothesis comes from Unsworth and Robison (2015), who examined individual differences in WMC and pupillary responses during a visual change detection task. Unsworth and Robison (2015) found that pupil dilation increased with increases in set size in a visual arrays working memory task. Specifically, we found that the pupil increased up until around four items and then plateaued for larger set sizes suggesting pupil dilation tracked attention allocation up to the capacity limits of working memory (see Alnaes et al. 2014 for similar results in multi-object tracking which tends to correlate with WMC; Drew \& Vogel, 2008). Unlike Heitz et al. (2008; see also Tsukahara et al., 2016) we found that variability in baseline pupil diameter, rather than mean pupil diameter, correlated with individual differences in WMC. We interpreted these results as suggesting that trial-to-trial fluctuations in task engagement (or lapses of attention) partially account for individual differences in WMC as indexed by pupillary responses. Thus, whereas Heitz et al. (2008) found evidence that low WMC individuals have smaller baseline pupil diameters (associated with hypoactive LC-NE functioning), Unsworth and Robison (2015) found that individual differences in WMC were related to variability in baseline pupil diameter suggesting that variability in LC-NE functioning was related to WMC. From these prior studies it is not clear what the relation (if any) is between baseline pupil diameter (as an index of LC-NE functioning) and WMC. Furthermore, as noted previously, another possibility is that there is no relation between LC-NE functioning and individual differences in $\mathrm{WMC}$ and attention control. In terms of pupillary responses this would suggest no relation between WMC and pupil diameter (both pre-trial baseline and TEPRs).

To better assess possible relations between WMC and LCNE functioning via pupil diameter, we (Unsworth \& Robison, 2016b) recently ran a larger scale latent variable pupillometry study. In this study, 165 participants performed multiple WMC (i.e., operation span, symmetry span, reading span) and attention control (i.e., antisaccade, Stroop, psychomotor vigilance) tasks. During the attention control tasks we assessed individual differences in off-task thoughts (i.e., mind-wandering, inattention, and external distraction) by periodically presenting participants with thought-probes to ascertain their current attentional state. Importantly, during the attention control tasks we simultaneously recorded pupil diameter as an index of arousal and LC-NE functioning. Consistent with prior pupillometry studies of mind-wandering, we found that when participants reported being off-task both their baseline pupil diameter and their TEPRs were smaller than when they reported being on task (Grandchamp et al., 2014; Mittner et al., 2014; Unsworth \& Robison, 2016c).

In terms of individual differences we found that variability in pupillary responses (standard deviation of both baseline and TEPRs) was related to individual differences in WMC and attention control. For example, shown in Fig. 6 are baseline pupil diameter (Fig. 6a) and TEPRs (Fig. 6b) across trials for one typical high WMC and one typical low WMC individual on the psychomotor vigilance task. As can be seen, the low WMC individual has more trial-to-trial fluctuations in both baseline pupil diameter and TEPRs.

Furthermore, variability in baseline pupil diameter and variability in TEPRs were highly related and the shared variance seemed to be critically important for the relation with WMC, attention control, and off-task thinking. For example, shown in Fig. 7a is a reanalysis of Unsworth and Robison (2016b). In this reanalysis we examined a confirmatory factor analysis of WMC, attention control, off-task thinking, and variability in pupil responses. As can be seen, similar to prior studies, WMC was related to attention control and to off-task thinking. Importantly, all of the measures of pupil variability (standard deviation of baseline and TEPRs from both the psychomotor vigilance task and the Stroop task) loaded on the same latent factor and this factor was related to latent factors of WMC, attention control, and off-task thinking. Note we allowed the error variances for the pupil measures from the same task correlate given strong task-specific residual relations. The fit of the model was acceptable, $\chi^{2}(57)=124.75, p<.01$, RMSEA $=.08$, NNFI $=.91$, CFI $=.93$, SRMR $=.07$. These results demonstrate that fluctuations in pupillary responses and potential variability in LC-NE functioning is related to individual differences in WMC and attention control.

Next we specified a structural equation model to examine the notion that individual differences in LC-NE fluctuations are related to individual differences in task-disengagement, which in turn are related to individual differences in attention control and WMC. Specifically, we specified a model in which individual differences in variability in pupillary responses are related to individual differences in off-task thoughts (task-disengagement), which are in turn related to individual differences in attention control and WMC. The fit of the model was acceptable, $\chi^{2}(60)=128.36, p<.01$, RMSEA $=.08$, NNFI $=.92$, CFI $=.94$, SRMR $=.08$. Figure $7 \mathrm{~b}$ shows the resulting structural equation model. As can be seen, variability in pupillary responses was associated with off-task thoughts, and these off-task thoughts in turn predicted individual differences in attention control and WMC. These results suggest that moment-to-moment fluctuations in LC-NE functioning predict individual differences in off-task thinking, attention control, and WMC. Those individuals who have more fluctuations in LC-NE functioning are more susceptible to attentional capture from internal 
(a)

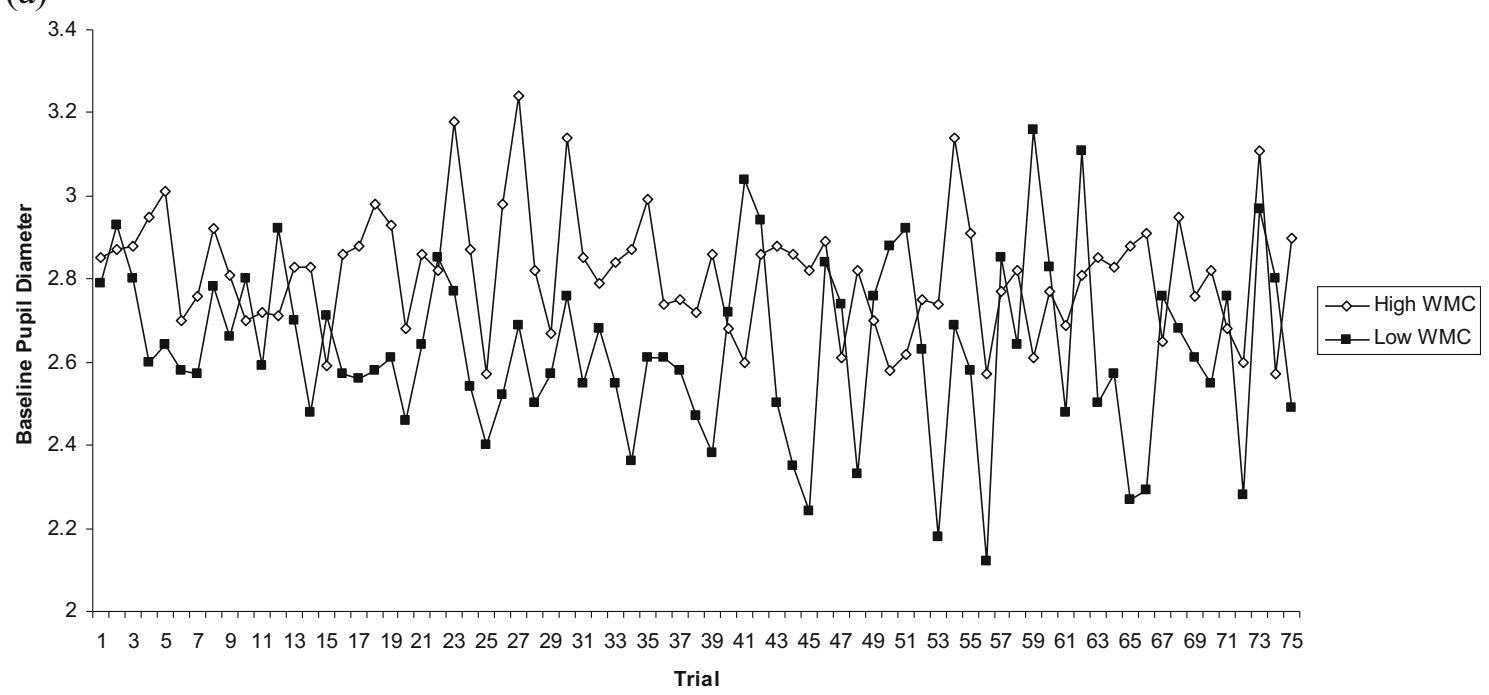

(b)

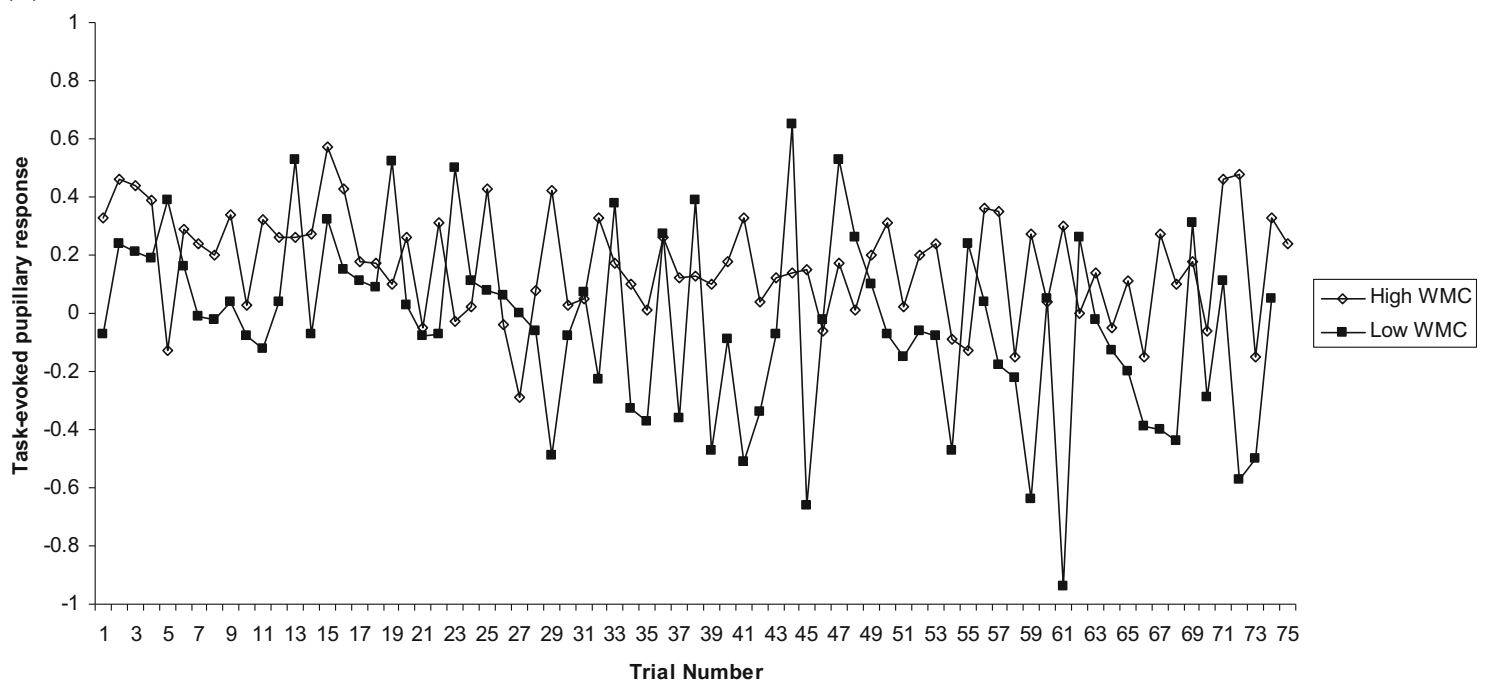

Fig. 6 (a) Trial-by-trial baseline pupil diameter for a typical high and typical low working-memory capacity (WMC) individual on the psychomotor vigilance task. (b) Trial-by-trial task-evoked pupillary

distraction (mind-wandering) and have lower overall attention control and WMC abilities. Along with prior research which has examined individual differences in WMC and attention control and pupillary responses these results suggest an important link between these constructs and further suggest that variation in LC-NE functioning may be an important piece to the WMC-attention control puzzle.

\section{Discussion}

In this article we have presented an account of individual differences in WMC and attention control suggesting that these individual differences partially reflect differences in LC-NE functioning. According to the current account the relation response for a typical high and typical low WMC individual on the Stroop task. Data from Unsworth \& Robison (2016b)

between $\mathrm{WMC}$ and attention control is partially due to the fact that low WMC is associated with LC dysregulation and attention control deficits due to an inability of the LC to properly and consistently modulate the amount of control exerted by the FPN. The main points may be summarized as follows.

1) WMC is related to attention control and attentional failures in and out of the laboratory.

2) Much of the relation between WMC and attention control seems to be due to fluctuations or lapses of attention. When on-task and focused, high and low WMC individuals show very similar levels of performance. However, low WMC individuals demonstrate more off-task thinking (e.g., mind-wandering) and task disengagement than high WMC individuals. 
(a)

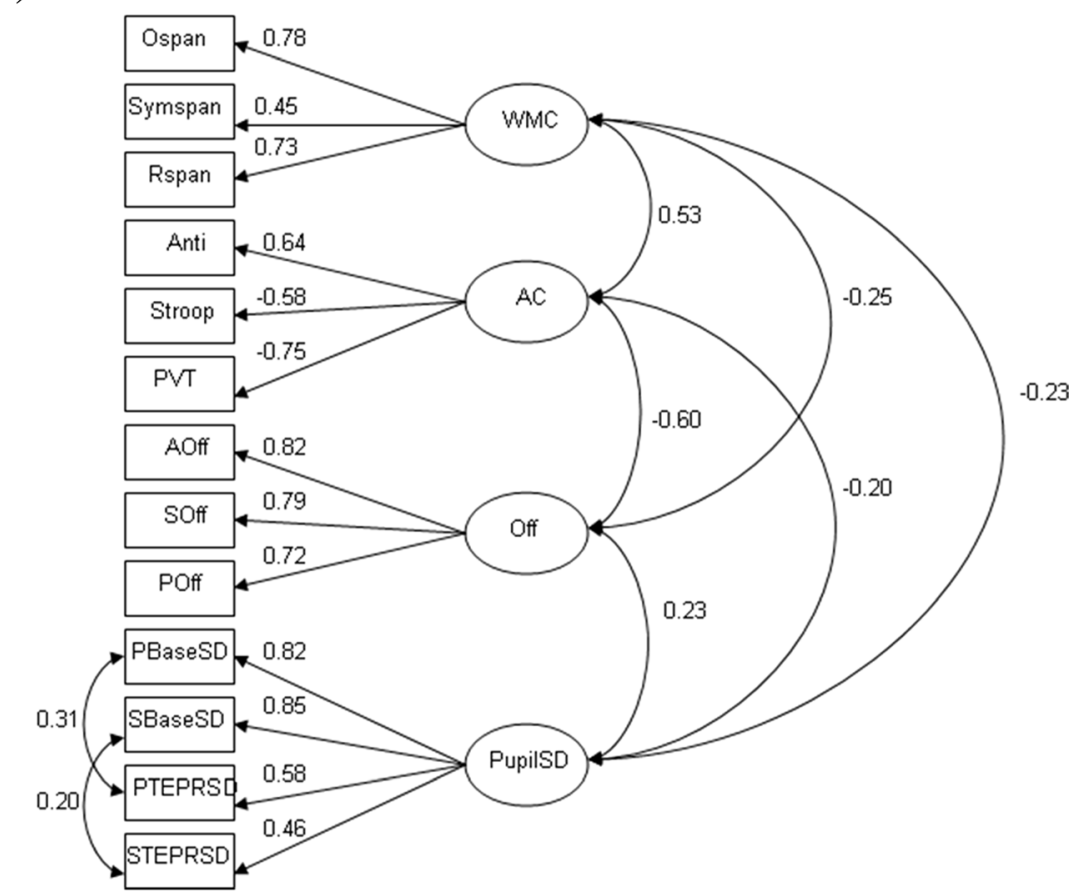

(b)

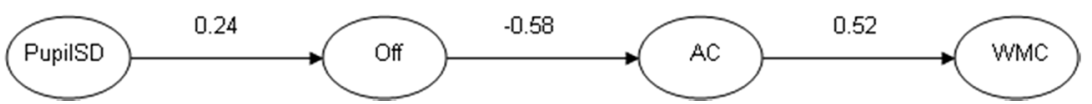

Fig. 7 (a) Confirmatory factor analysis model for working memory capacity (WMC), attention control (AC), off-task thinking (Off), and variability in pupillary responses (PupilSD). Paths connecting latent variables (circles) to each other represent the correlations between the constructs and the numbers from the latent variables to the manifest variables (squares) represent the loadings of each task onto the latent variable. (b) Structural equation model in which variability in pupillary responses predict off-task thinking, which predicts attention control, and attention control predicts working memory capacity. All paths are significant at the $p<.05$ level. Ospan operation span, Rspan reading

3) The LC-NE system is important for regulating attentional state in a variety of situations. In particular, the LC is thought to modulate the FPN (which in turn suppresses DMN) to focus attention on important external information (the current task). Trial-to-trial changes in the LC result in trial-to-trial changes in the FPN. When the FPN is weakly activated, the DMN is not suppressed, resulting in task disengagement and off-task thinking. Upon awareness of being off-task (potentially due to poor task performance) the SN signals the LC to bring performance back on line.

4) Variation in $\mathrm{WMC}$ and attention control is partially due to dysregulation of LC-NE functioning. Because of dysregulation of LC-NE functioning, the FPN for low WMC individuals is only weakly activated, resulting in greater DMN activity (and greater mind-wandering) for low WMC individuals compared to high WMC individuals. This results in disrupted attention control and overall span, Symspan symmetry span, Anti antisaccade, Stroop color word Stroop task, PVT psychomotor vigilance task, Aoff off-task thoughts Antisaccade, Soff off-task thoughts Stroop, Poff off-task thoughts psychomotor vigilance task, PBaseSD standard deviation of baseline pupil diameter psychomotor vigilance task, SBaseSD standard deviation of baseline pupil diameter Stroop, PTEPRSD standard deviation taskevoked pupillary response psychomotor vigilance task, STEPRSD standard deviation task-evoked pupillary response Stroop. Data from Unsworth and Robison (2016)

more erratic performance (more lapses of attention) for low WMC individuals than for high WMC individuals. This LC dysregulation can take on multiple forms (too little tonic LC activity, too much tonic LC activity, too many fluctuations between different LC modes). Although many prior studies suggest individual differences in WMC are due to differences in FPN, these differences may actually be a consequence of disrupted LC functioning.

5) Examining pupillary responses as a correlate of LCNE functioning suggests that individual differences in WMC and attention control are primarily related to fluctuations in pupillary responses (both baseline and TEPRs) rather than differences in baseline/tonic levels. These latent variable results are consistent with the notion that moment-to-moment fluctuations in LC$\mathrm{NE}$ functioning are related to differences between individuals in $\mathrm{WMC}$ and attention control. 
This framework represents an extension of executive attention and attention control views of WMC which suggest that a core aspect of WMC is the ability to control attention to ensure that task-relevant representations are maintained in an active state in the presence of interference and distraction (e.g., Engle \& Kane, 2004; Kane \& Engle, 2002; Unsworth \& Engle, 2007). Here we suggest a possible reason for individual differences in WMC and attention control in the form of differences in LC-NE functioning. This framework is able to account for many prior findings relating WMC to attention control in terms of task performance, self-reports of mind-wandering, and neural correlates. Furthermore, this framework links individual differences in WMC to individual differences in physiological correlates of LC-NE functioning such as pupil diameter (e.g., Heitz et al., 2008; Unsworth \& Robison, 2015) which were further corroborated in a new large-scale latent variable study. Future studies relying on combinations of individual differences, genetics, fMRI, psychopharmacological, and physiological (pupillometry, EEG) correlates of putative LC-NE functioning are needed to further test and refine the current ideas. As such, the current theory provides a powerful framework in which to interpret individual differences in WMC and attention control and provides a number of promising future directions within which to examine individual differences in WMC and attention control.

\section{Possible heterogeneity of WMC and LC-NE functioning}

In linking LC-NE functioning to individual differences in WMC and attention control we hypothesized three ways in which high and low WMC individuals may differ. Specifically, we suggested that low WMC individuals may have lower tonic LC levels than high WMC individuals, resulting in low WMC individuals being in a constant hypoactive mode. This would result in the LC not effectively modulating the FPN and reduced attention control abilities. Another possibility suggested was that low WMC individuals have overall too high tonic LC levels than high WMC individuals resulting in low WMC individuals being in a constant hyperactive mode. This too would result in disrupted FPN activity and reduced attention control abilities. Finally, we also suggested the possibility that low WMC individuals fluctuate between different states more readily than high WMC individuals. This would result in more inconsistent attention control abilities across trials. Thus, LC dysregulation can take on multiple forms. Our latent variable pupillary results strongly suggested that low WMC individuals experienced more fluctuations in LC modes than high WMC individuals (see also Unsworth \&
Robison, 2015), and these fluctuations are related to the propensity for off-task thinking and lowered performance on attention control tasks. Thus, this would suggest that the source of low WMC individuals' LC-NE disrupted functioning is due to fluctuations in tonic activity rather than overall differences in tonic levels. However, we would be remiss not to point out that it is entirely probable that high and low levels of tonic LC activity are also contributing factors for some low WMC individuals. That is, it is likely that there is heterogeneity in WMC and LC-NE functioning such that some low WMC individuals have lower tonic activity and some low WMC individuals have higher tonic activity. Within the current sample of relatively highfunctioning university students, differences seemed to be due to differences in fluctuations between LC modes. However, with other samples of individuals, different results could occur. For example, in three experiments using a mixed sample of university students and community volunteers, Heitz et al. (2008; see also Tsukahara et al., 2016) found that low WMC individuals had consistently smaller baseline pupil diameters than high WMC individuals. Thus, this suggests that in some samples of individuals, differences can arise from low WMC individuals having lower tonic LC activity levels resulting in lowered arousal and alertness. Additionally, it is possible that some low WMC individuals have attentional problems due to too much LC tonic activity resulting in over-activity of the FPN. This could result from test anxiety or from personality traits such as neuroticism, which could lead to higher levels of stress associated with increased tonic LC activity (Millan, 2003; Szabadi, 2013). Indeed, in Unsworth and Robison (2016b) we found a negative relationship between baseline pupil diameter and WMC suggesting that low WMC individuals had larger baselines than high WMC individuals (opposite to Heitz et al., 2008). As part of another project carried out in our laboratory at the same time, 119 participants also completed a personality questionnaire in which neuroticism was assessed (Robison et al., in press). Combining that data with the Unsworth and Robison (2016b) data suggests that neuroticism was related to WMC $(r=-.34)$ and to baseline pupil diameter $(r=$ .22). Importantly, partialling out neuroticism from the WMC-baseline pupil diameter relation resulted in a significantly weaker correlation between WMC and baseline pupil diameter $(r=-.16)$. Thus, within the current sample of participants, part of the reason for the negative relation between WMC and baseline pupil diameter seemed to be due to shared variance with neuroticism. An important aspect of future research will be to examine the extent to which heterogeneity in WMC and attention control is a result of different subgroups of low WMC individuals who differ in LC-NE functioning as indicated by variability in baseline pupil diameter. 


\section{Other sources of variability in WMC and attention control}

In the current account we have primarily focused on goal maintenance abilities in which lapses of goal maintenance (goal neglect) occur when LC tonic activity is too high or too low on a trial-by-trial basis. However, it is important to recognize that there are likely other sources of variability contributing to the overall WMC-attention control relation. That is, several theories suggest that goal maintenance abilities are just one reason why WMC and attention control are related. For example, in their prominent two-factor theory of WMC and cognitive control, Kane and Engle (2003; Engle \& Kane 2004) have suggested that in addition to goal maintenance abilities, conflict resolution is also an important factor. According to Kane and Engle (2003), even when the task goal is properly maintained, low WMC individuals should still have problems resolving conflict that arises between the task goal and more habitual behaviors. For example, in the Stroop task, conflict resolution abilities are needed to resolve the conflict between the goal (say the color) and habitual word reading even in the absence of goal neglect. Evidence in support of this is the finding that low WMC individuals demonstrate more Stroop interference than high WMC individuals even on tasks where mostly incongruent trials are presented (Kane \& Engle, 2003; Meier \& Kane, 2013). It is argued that on these types of trials, constantly being presented with incongruent stimuli serves to reinforce the goal and reduce goal maintenance requirements. However, differences between high and low WMC individuals still arise due to the fact that low WMC individuals are poorer at resolving conflict and competition than high WMC individuals. Recently Meier and Kane (2015) examined the relation between WMC and conflict resolution in versions of the Simon task. Meier and Kane found that situations that minimized goal maintenance needs, low WMC individuals experienced more stimulusstimulus conflict than high WMC individuals, suggesting that WMC is related to conflict resolution during selection. Thus, we acknowledge that an additional source of variability in WMC and attention control is conflict resolution abilities that may be particularly poor for some low WMC individuals. At the same time we note that evidence in support of conflict resolution differences tend to be mixed and somewhat weaker than evidence for goal maintenance abilities. For example, one prediction of the conflict resolution account is that on incongruent trials high and low WMC individuals should demonstrate different RT distributions with low WMC individuals having a distribution in which the mean is shifted toward slower responses than high WMC individuals (Kane \& Engle, 2003). That is, conflict resolution is a constant timeconsuming process (Kane \& Engle, 2003). However, when examining WMC differences for incongruent RT distributions some results suggest a difference in the mean on some tasks
(Meier \& Kane, 2015; Unsworth et al., 2012) but not on others (Meier \& Kane, 2015; Unsworth et al., 2012). Furthermore, Morey et al. (2012) found that only versions of the Stroop with a high proportion of congruent trials correlated with WMC. When all incongruent trials were presented or a cross-modal Stroop task was used, WMC did not correlate with performance. Morey et al. concluded that WMC is related to goal maintenance abilities but not conflict resolution abilities. As noted by Kane and Engle (2003) these mixed results could arise because differences in RTs between high and low WMC individuals in conflict resolution situations may be masked and thus require a large number of trials to find a somewhat small effect. Furthermore, as noted by Meier and Kane (2015) not all manifestations of conflict resolution are related to WMC. Thus, more work is needed to better delineate the relation between $\mathrm{WMC}$ and conflict resolution abilities.

In a similar vein, Braver, Gray, and Burgess (2007; see also Braver, 2012) have proposed a dual-mechanism account of cognitive control. In this account cognitive control is broken down into two modes: proactive control and reactive control. Proactive control refers to active maintenance abilities in which goal representations and context are maintained in an active state to ensure active task performance. Whereas proactive control is engaged prior to the presentation of a stimulus, reactive control is engaged after a stimulus has been presented. Reactive control serves to resolve conflict in the moment by reactivating the task goal or context. Thus, like the two-factor theory proposed by Kane and Engle (2003; Engle $\&$ Kane, 2004), this view suggests that both proactive active maintenance abilities and reactive conflict resolution abilities are important for performance on various tasks and for individual differences in performance. A series of recent studies by Redick and colleagues (Redick \& Engle, 2011; Redick, 2014; Richmond, Redick, \& Braver, 2015) have examined the extent to which individual differences in WMC can be accounted for by the dual-mechanisms account using variations of the AX-CPT task. In particular, this research suggests that high WMC individuals are better at utilizing a proactive control strategy to prepare the correct action than low WMC individuals. That is, high WMC individuals more consistently engage proactive control than low WMC individuals. Low WMC individuals rely more on reactive control. Consistent with the current proposal, this difference in proactive control could arise due to periodic lapses of attention in which the task goal is lost from working memory and low WMC individuals are more likely to have to rely on reactive control on those trials than high WMC individuals, who are less likely to experience a lapse of attention. That is, a sufficient amount of arousal may be needed to engage in proactive control/ preparatory attention, and when arousal fluctuates, one must rely on reactive control to perform the task. Furthermore, similar to the two-factor theory proposed by Kane and Engle (2003), this account suggests that in situations where 
proactive control is unlikely, high WMC individuals will be better at implementing reactive control than low WMC individuals. Similar to conflict resolution, we acknowledge the possibility that reactive control is another potent means of control that is likely related to WMC especially in certain contexts.

In addition to differences in conflict resolution and reactive control, we suggest that there are other potential ways in which WMC is related to attention control abilities. For example, examining Fig. 4 suggests that individual differences in WMC and attention control result from differences in LC functioning which in turn causes differences in FPN (and $\mathrm{DMN})$. However, it is also possible that variation in the other core brain networks give rise to some individual differences in WMC as well. For example, it is possible that some low WMC individuals do not have LC problems, but rather have reduced attention control abilities due to disruption of the FPN directly. These individuals would demonstrate reduced attention control abilities in a variety of tasks even when goal maintenance and proactive control are not necessary (but conflict resolution and reactive control are). Furthermore, these individuals may experience broader deficits in attention control such as inabilities to configure attention to particular objects or spatial locations (Bleckley, Durso, Crutchfield, Engle, \& Khanna, 2003; Bleckley, Foster, \& Engle, 2015). Furthermore, these individuals may have particular problems filtering out irrelevant information and preventing attentional capture (Fukuda \& Vogel, 2009, 2011; Vogel, McCollough, \& Machizawa, 2005), which may be unrelated to lapses of attention and mind-wandering. For example, in a recent study we found that both mind-wandering and filtering predicted WMC, but that mind-wandering and filtering were unrelated and accounted for separate sources of variance in WMC (Unsworth \& Robison, 2016a). Thus, although we suggest that LC dysregulation drives much of the WMC-attention control relation, it is likely that some of the WMC-attention control relation stems from variability in FPN functioning rather than variability in LC-NE functioning.

Similarly it is possible that variability in DMN activity accounts for some of the WMC-attention control relation and accounts for some variability in mind-wandering. That is, rather than LC dysregulation contributing to lowered attention control via FPN, it is possible that some individuals have disturbances in DMN with an overactive DMN that causes frequent intrusions of self-generated thoughts and mindwandering which impede successful task performance. This is consistent with the default-mode interference hypothesis proposed by Sonuga-Barke and Castellanos (2007) to account for lapses of attention and performance variability seen in ADHD. It is possible that some of the WMC-attention control relation is due to variability in the strength of the DMN, with some low WMC individuals experiencing periodic lapses of attention and mind-wandering because the DMN is interfering with task performance. On tasks requiring focused attention to external events, this would result in lowered attention control and lowered task performance. However, in tasks requiring introspective self-generated thoughts this could actually lead to enhanced performance. Thus, some of the variability shared between WMC and mind-wandering may be a direct result of DMN regulation rather than LC-NE functioning.

Finally, it seems possible that some of the WMC-attention control relation is due to deficits in the $\mathrm{SN}$ which results in compromised monitoring and subsequent adjustments in control. Some low WMC individuals may have specific problems monitoring their performance and signaling the LC to get back on task and increase control in the FPN. That is, if you don't know you are experiencing a lapse of attention or you aren't aware of the errors you are making, then subsequent adjustments in control will not occur and performance will continue to suffer. If this is the case then one would expect WMC to be related to classic indicators of conflict monitoring and microadjustments of control such as post-error slowing and congruency sequence effects (e.g., Botvinick et al., 2001). However, the evidence is decidedly mixed. For example, in four experiments Unsworth et al. (2012) found no evidence for WMC differences in post-error slowing on a number of attention control tasks. Miller, Watson, and Stayer (2012) found that high WMC individuals demonstrated a larger error-related negativity and post-error positivity than low WMC individuals (although there were no differences in behavioral performance). Furthermore, Unsworth et al. (2012) found no relation between congruency sequence effects in Stroop and flanker tasks and WMC (see also Keye et al., 2009; Meier \& Kane, 2013), and Meier and Kane (2015) found no relations in versions of the Simon task. However, Keye et al. (2009) and Weldon et al. (2013) did find significant relations in versions of the Simon task. Furthermore, Gulbinaite et al. (2014), despite finding no behavioral relations, found significant WMCcongruency sequence effects in EEG oscillations. Thus, several studies find no relations between behavioral markers of conflict monitoring and micro-adjustments of control, but some studies find some evidence for physiological relations with WMC.

Collectively there seem to be somewhat mixed results suggesting WMC relations with conflict resolution and conflict and error monitoring (both behavioral and physiological). These mixed results could be due to relatively weak and non-stable effects, or they could be due to heterogeneity in WMC and attention control. That is, there are a number of ways to score low on WMC measures and perform poorly on measures of attention control. It is entirely possible that there are sub-groups of low WMC individuals (Unsworth, 2009; Unsworth et al., 2014). Some low WMC individuals may have issues with goal maintenance, some have issues with conflict resolution, and some have issues with conflict and error monitoring. Individuals with goal maintenance 
issues may be the predominant type of low WMC individuals, with smaller subgroups having deficits in conflict resolution and/or conflict and error monitoring. Future research is needed to better delineate the many possible sources of variability linking WMC and attention control and future research is needed to better examine potential subgroup variation in $\mathrm{WMC}$ and attention control.

\section{The role of the LC-NE in other working memory facets}

According to the multifaceted view of WMC, there are multiple different facets on which individuals differ (e.g., Unsworth, 2016; Unsworth et al., 2014). As noted previously, we have suggested that individual differences in WMC arise not only from differences in attention control abilities, but also from differences in primary memory capacity (the number of items that can be simultaneously maintained in an active state) and differences in secondary memory (both encoding and retrieval from secondary memory). Furthermore, we have suggested that all three facets are critically important in accounting for the relation between WMC and higher-order cognition (such as fluid intelligence). In the current paper we have mainly focused on differences in attention control, but it is important to note that these three facets interact and thus, LC-NE functioning will also influence primary memory capacity and secondary memory abilities. For example, as suggested by Kahneman (1973), capacity is determined in part by current arousal levels. Thus, when arousal is optimal, capacity will be at its maximum, but when arousal is too high or too low capacity will be reduced, leading to reductions in performance (Kahneman, 1973). This suggests that capacity will be determined in part via current LC tonic levels which modulate the FPN (e.g., Usher \& Davelaar, 2002). Individuals with too low or too high tonic LC levels will tend to have compromised capacities and overall lower estimates of capacity than individuals with more optimal tonic LC levels. As noted previously, prior research has shown that TEPRs accurately track the number of items being maintained such that TEPRs during a delay in a change detection task increased until around four items and then plateaued consistent with behavioral estimates of capacity (Unsworth \& Robison, 2015). Examining individual differences we found that behavioral estimates of capacity correlated with TEPRs $(r=.43)$, suggesting that high WMC individuals TEPRs plateaued at higher values than low WMC individuals. Additionally, individuals who experience more fluctuations in tonic LC levels will also tend to have reduced estimates of capacity given that they will be more likely to experience trial-to-trial fluctuations in capacity which will result in overall lower average capacity estimates. Indeed, recent research has suggested that lowered capacity estimates seen in low WMC individuals are partially due to differences in lapses of attention (Adam et al., 2015; Unsworth \& Robison, 2016a). Thus, differential LC-NE functioning can impact not only overall attention control abilities, but also an individual's ability to simultaneously maintain several representations in an active state for on-going processing.

Differences in LC-NE functioning should also influence individual differences in secondary memory abilities. For example, the LC has direct projections to the hippocampus (Samuels \& Szabadi, 2008a) and it has been suggested that the LC is critically important for memory formation and the notion that arousal enhances memory representations (McGaugh, 2006). The importance of the LC in memory formation may be partially due to attentional modulation of hippocampal neurons (Rowland \& Kentros, 2008). Thus, the LCNE system may be particularly important for modulating the intensity of attention to items during encoding, which results in stronger hippocampal representations that are then easier to retrieve at recall. Thus, high WMC individuals may have better secondary memory abilities than low WMC individuals, in part due to differences in LC-NE functioning such that they are better able to modulate the amount of attention to items at encoding resulting in stronger representations that are more readily available during retrieval. Likewise, high WMC individuals may be more able to modulate attention during retrieval than low WMC individuals to better select target representations amongst competitors during especially challenging retrieval situations (such as when proactive or retroactive interference are present). Again this suggests the likely importance of the LC-NE system not only for individual differences in attention control, but also the interactions amongst various facets of the overall working memory system. Future research is needed to better examine the interactions between these important components of working memory and the possible influence of the LC-NE system to each component individually as well as interactions among the different components.

\section{Other neuromodulatory influences on WMC and attention control}

In the current work we have mainly focused on the LC-NE system given its importance for regulating attentional state and fluctuations in attention control which seem pivotal to individual differences in WMC and attention control. However, it is also important to note that other neuromodulators also likely influence WMC and attention control in potentially different ways. Here we briefly consider possible influences of dopamine and acetylcholine on individual differences in WMC and attention control, while acknowledging that there are complex associations and interactions among these various neurotransmitters. It is beyond the scope of the current paper to examine the full complexities of these interactions and relations. 
Dopamine has long been seen as an important neuromodulator of the FPN and linked to overall working memory functioning and attention control (Robbins \& Arnsten, 2009). Like NE, dopamine is thought to modulate signal to noise ratios in target neurons (gain modulation) in response to salient events (in particular motivationally salient events) leading to an increase in alertness (Bromberg-Martin, Matsumoto, \& Hikosaka, 2010; Servan-Schreiber et al. 1990). Dopamine demonstrates a similar inverted U-function with performance and FPN functioning (e.g., Robbins \& Arnsten, 2009). Classically, NE was associated with overall arousal levels and dopamine with reward processing. More recent research suggests that dopamine is critically important for updating the contents of working memory via an adaptive gating mechanism (e.g., Cohen, Aston-Jones, \& Gilzenrat, 2004). In this account, dopamine acts to gate input to the FPN allowing the contents of working memory to be updated in a selective manner. It is assumed that gating occurs via reinforcement learning such that gating occurs when there is an opportunity for reward ensuring that in future reward contexts gating and updating is more likely to occur (D'Ardenne et al., 2012). Furthermore, dopamine may be particularly important for decision making regarding the costs and benefits of engaging in effortful cognitive activities (Westbrook \& Braver, 2016). For example, Varazzani et al. (2015) demonstrated that dopaminergic neurons in the substantia nigra were sensitive to expected reward and the cost of engaging in effortful processes, whereas NE neurons in the LC were sensitive to effort production in order to energize behavior. These LC-NE responses were significantly related to pupil dilation during task performance. Dopamine seems critically important for not only ensuring active maintenance of goal states in the FPN, but also for updating the contents of working memory via reinforcement learning and decision making of under what circumstances the allocation of effort is worthy, whereas the LC-NE system may be more important for allocating resources and energizing behavioral responses. Thus, dopamine and NE are both likely important for working memory and attention control, but they may be associated with different aspects of attention control. Furthermore, in terms of individual differences, dopamine is likely important for the active maintenance of task goals seen as a hallmark of individual differences in WMC. As noted previously, prior work suggests that individual differences in WMC are linked with proactive control mechanisms that are thought to be mediated via phasic dopamine activity (Braver, 2012; Braver et al., 2007; Redick, 2014; Richmond, Redick, \& Braver, 2015). Furthermore, it is possible that low WMC individuals have deficits in reinforcement learning resulting in inaccurate timing of gating signals such that irrelevant stimuli (internal or external) are allowed to gain access to working memory. Thus, dopamine is also likely critically important for individual differences in WMC and attention control. The influence of dopamine on individual differences in WMC and attention control may be independent of NE, but it is also likely that these two systems interact in important ways. For example, there are strong reciprocal connections between the LC and the ventral tegmental area (Chandler, Waterhouse, \& Gao, 2014). Thus, given the highly interactive nature of these two neuromodulatory systems it will be important to not only understand how they potentially independently account for variation in WMC and attention control, but also their joint effects in terms of computing effort costs and for energizing effortful behaviors.

Additionally, it is likely possible that other neuromodulators such acetylcholine are important for WMC and attention control. Prior research has suggested that the FPN is innervated by cholinergic neurons and that these neurons are especially important for attentional functions (Hasselmo, \& Sarter, 2011) and in particular for attention control (Sarter \& Paolone, 2011). In particular, Sarter and colleagues (2005) have suggested that acetylcholine is critically important for attentional effort. They suggest that attentional effort is needed "to maintain or regain attentional performance under challenging conditions" (p. 147). When performance begins to decline or it is difficult to sustain attention to the current task, it is assumed that cholinergic activity increases in the FPN resulting in an enhancement of targets and a suppression of distractors that allows performance to either maintain current levels or recover if performance has decreased. In this view acetylcholine plays a similar role as $\mathrm{NE}$ in regulating attentional state particularly during challenging attentional tasks or following shifts in performance (such as recovering performance following an error or a mind-wandering episode). Thus, this attentional effort system seems critically important in challenging situations where "negative events signal that goals are not being achieved, making effort necessary" (Sarter et al., 2006, p. 151). Thus, whereas dopamine may be important for evaluating the effort costs, and NE may be important for energizing effortful processes, acetylcholine may be additionally important in situations where effortful processing has declined and a further booster of attentional effort is needed to get performance back on track. As such, dysregulation of this system would likely result in decreased attention control, increased mind-wandering, and overall erratic performance as attention is likely to be captured by internal and external distractors similar to what is seen in low WMC and attention control individuals. Furthermore, pupillary responses are controlled by both the sympathetic and parasympathetic nervous systems. The dilator pupillae is innervated by adrenergic input from the sympathetic nervous system resulting in dilation, whereas the sphincter pupillae is innervated by cholinergic input from the parasympathetic nervous system resulting in constriction. Thus, pupil dilation can occur via a complex interaction of the sympathetic and parasympathetic nervous systems. Although most work has 
examined pupil dilation in response to attentional demands via sympathetic activation from the LC-NE, it is also important to examine potential cholinergic factors related to attentional effort and pupillary responses. Thus, future work should examine the importance of strong interactions between NE and acetylcholine in relation to moment-to-moment fluctuations in pupillary responses (e.g., Reimer et al., 2016) and attentional state (Brown et al., 2016). Collectively, prior research suggests an important role of acetylcholine in regulating attentional effort, and dysregulation of this system likely results in reduced attention control and WMC. Future research is needed to better clarify how these neuromodulatory systems interact and their possible roles in accounting for individual differences in WMC and attention control abilities.

\section{Concluding remarks}

In this article we have advanced the view that variability in LC-NE functioning is partially responsible for individual differences in WMC and attention control. Given its wide projections throughout the neocortex, the LC-NE system is important for modulating information and neural processing in different networks. In particular, the LC-NE seems critically important for modulating attention control and working memory demands in the FPN. In terms of individual differences, we suggest that low WMC is associated with LC dysregulation which results in more moment-to-moment fluctuations in tonic LC activity, fluctuations in phasic LC responses, increased off-task thinking, and lowered attention control compared to high WMC. Combining experimental, differential, computational, and psychophysiological programs provides a promising means for explaining and understanding cognitive and physiological differences among individuals and how these differences give rise to variation in higher-order cognitive processes.

Author notes The authors thank Randy Engle for initially inspiring this line of research.

This research was supported by Office of Naval Research grant N00014-15-1-2790.

\section{References}

Adam, K. C. S., Mance, I., Fukuda, K., \& Vogel, E. K. (2015). The contributions of attentional lapses to individual differences in working memory capacity. Journal of Cognitive Neuroscience, 8, 1601-1616.

Alnaes, D., Sneve, M. H., Espeseth, T., Endestad, T., van de Pavert, S. H. P., \& Laeng, B. (2014). Pupil size signals mental effort deployed during multiple object tracking and predicts brain activity in the dorsal attention network and the locus coeruleus. Journal of Vision, 14, 1-20.
Andrews-Hanna, J. R., Smallwood, J. S., \& Spreng, R. N. (2014). The default network and self-generated thought: Component processes, dynamic control, and clinical relevance. Annals of New York Academy of Sciences - Year in Cognitive Neuroscience Special Issue, 1316, 29-52.

Arnsten, A. F. (2009). Toward a new understanding of attention-deficit hyperactivity disorder pathophysiology. CNS Drugs, 23, 33-41.

Arnsten, A. F., \& Goldan-Rakic, P. S. (1984). Selective prefrontal cortical projections to the region of the locus coeruleus and raphe nuclei in the rhesus monkey. Brain Research, 306, 9-18.

Aston-Jones, G., \& Cohen, J. D. (2005). An integrative theory of locus coeruleus-norepinephrine function: Adaptive gain and optimal performance. Annual Review of Neuroscience, 28, 403-450.

Aston-Jones, G., Iba, M., Clayton, E., Rajkowski, J., \& Cohen, J. (2007). The locus coeruleus and regulation of behavioral flexibility and attention: Clinical implications G.A. Ordway, M.A. Schwartz, A. Frazer (Eds.), Brain norepinephrine: Neurobiology and therapeutics, Cambridge University Press, pp. 196-235.

Baddeley, A. D. (1993). Working memory or working attention? In A. Baddeley \& L. Weiskrantz (Eds.), Attention: Selection, awareness, and control (pp. 152-170). Oxford: Oxford University Press.

Beatty, J. (1982a). Task-evoked pupillary responses, processing load, and the structure of processing resources. Psychological Bulletin, 91, 276-292.

Beatty, J. (1982b). Phasic not tonic pupillary responses vary with auditory vigilance performance. Psychophysiology, 19, 167-172.

Beatty, J., \& Lucero-Wagoner, B. (2000). The pupillary system. In J. T. Cacioppo, L. G. Tassinary, \& G. G. Berntson (Eds.), Handbook of psychophysiology (pp. 142-162). New York: Cambridge University Press.

Beilock, S. L., \& Carr, T. H. (2005). When high-powered people fail: Working memory and "choking under pressure" in math. Psychological Science, 16, 101-105.

Berridge, C. W., \& Waterhouse, B. D. (2003). The locus coeruleusnoradrenergic system: Modulation of behavioral state and statedependent cognitive processes. Brain Research Reviews, 42, 33-84.

Bleckley, M. K., Durso, F. T., Crutchfield, J. M., Engle, R. W., \& Khanna, M. M. (2003). Individual differences in working memory capacity predict visual attention allocation. Psychonomic Bulletin \& Review, 10, 884-889.

Bleckley, M. K., Foster, J. L., \& Engle, R. W. (2015). Working memory capacity accounts for the ability to switch between object-based and location-based allocation of visual attention. Memory \& Cognition, 43, 379-388

Bonnelle, V., Ham, T. E., Leech, R., Kinnunen, K. M., Mehta, M. A., \& Greenwood, R. J. (2012). Salience network integrity predicts default mode network function after traumatic brain injury. Proceedings of the National Academy of Sciences, U.S.A., 109, 4690-4695.

Botvinick, M. M., Braver, T. S., Barch, D. M., Carter, C. S., \& Cohen, J. D. (2001). Conflict monitoring and cognitive control. Psychological Review, 108, 624-652.

Braem, S., Coenen, E., Bombeke, K., van Bochove, M. E., \& Notebaert, W. (2015). Open your eyes to prediction errors. Cognitive Affective Behavioral Neuroscience, 15, 374-380.

Braver, T. S. (2012). The variable nature of cognitive control: A dual mechanisms framework. Trends in Cognitive Science, 16, 106-13.

Braver, T.S., Gray, J.R., \& Burgess, G.C. (2007). Explaining the many varieties of working memory variation: dual mechanisms of cognitive control. In A.R.A Conway, C. Jarrold, M.J. Kane, A. Miyake, \& J.N. Towse (Eds.), Variation in Working Memory. NY: Oxford.

Breeden, A.L., Siegle, G.J., Norr, M.E., Gordon, E.M., \& Vaidya, C.J. (in press). Coupling between spontaneous pupillary fluctuations and brain activity relates to inattentiveness. European Journal of Neuroscience. 
Bressler, S. L., \& Menon, V. (2010). Large-scale brain networks in cognition: Emerging methods and principles. Trends in Cognitive Sciences, 14, 277-290.

Bromberg-Martin, E. S., Matsumoto, M., \& Hikosaka, O. (2010). Dopamine in motivational control: Rewarding, aversive, and alerting. Neuron, 68, 815-834.

Brown, S. D., \& Heathcote, A. J. (2008). The simplest complete model of choice response time: Linear ballistic accumulation. Cognitive Psychology, 57, 153-178.

Brown, G. G., Kindermann, S. S., Siegle, G. J., Granholm, E., Wong, E. C., \& Buxton, R. B. (1999). Brain activation and pupil response during covert performance of the Stroop Color Word task. Journal of International Neuropsychological Society, 5, 308-319.

Brown, S. B., Slagter, H. A., van Noorden, M. S., Giltay, E. J., van der Wee, N. J., \& Nieuwenhuis, S. (2016). Effects of clonidine and scopolamine on multiple target detection in rapid serial visual presentation. Psychopharmacology, 233, 341-350.

Bunge, S. A., Klingberg, T., Jacobsen, R. B., \& Gabrieli, J. D. E. (2000). A resource model of the neural basis of executive working memory. Proceedings of the National Academy of Sciences, 97, 3573-3578.

Burgess, G. C., Conway, A. R. A., Gray, J. R., \& Braver, T. S. (2011). Neural mechanisms of interference control underlie the relationship between fluid intelligence and working memory span. Journal of Experimental Psychology: General, 140, 674-692.

Cai, W., Chen, T., Ryali, S., Kochalka, J., \& Menon, V. (2016). Causal Interactions within a Frontal-Cingulate-Parietal Network during Cognitive Control: Convergent evidence from a multisite-multitask investigation. Cerebral Cortex, 26, 140-153.

Chamberlain, S. R., \& Robbins, T. W. (2013). Noradrenergic modulation of cognition: Therapeutic implications. Journal of Psychopharmacology, 27, 694-718.

Chandler, D. J., Waterhouse, B. D., \& Gao, W. J. (2014). New perspectives on catecholaminergic regulation of executive circuits: Evidence for independent modulation of prefrontal functions by midbrain dopaminergic and noradrenergic neurons. Frontiers in Neural Circuits, 8, 53.

Chatham, C. H., Frank, M. J., \& Munakata, Y. (2009). Pupillometric and behavioral markers of a developmental shift in the temporal dynamics of cognitive control. Proceedings of the National Academy of Sciences, 106, 5529-5533.

Chee, M. W., Chow Tan, J., Zheng, H., Parimal, S., Weissman, D. H., Zagorodnov, V., et al. (2008). Lapsing during sleep deprivation is associated with distributed changes in brain activation. The Journal of Neuroscience, 28, 5519-5528.

Chein, J., Moore, A., \& Conway, A. (2011). Domain-general mechanisms of complex working memory span. NeuroImage, 54, 550-559.

Cheyne, J. A., Solman, G. J. F., Carriere, J. S. A., \& Smilek, D. (2009). Anatomy of an error: A bidirectional state model of task engagement/disengagement and attention-related errors. Cognition, $111,98-113$.

Chiew, K. S., \& Braver, T. S. (2013). Temporal dynamics of motivationcognitive control interactions revealed by high-resolution pupillometry. Frontiers in Psychology, 4, 15.

Cohen, R. L. (1994). Some thoughts on individual differences and theory construction. Intelligence, 18, 3-13.

Cohen, J. D., Aston-Jones, G., \& Gilzenrat, M. S. (2004). A systems-level perspective on attention andcognitive control: Guided activation, adaptive gating, conflict monitoring, and exploitation vs.exploration. In M. I. Posner (Ed.), Cognitive neuroscience of attention (pp. 71-90). New York: Guilford Press.

Colflesh, G. J. H., \& Conway, A. R. A. (2007). Individual differences in working memory capacity and divided attention in dichotic listening. Psychonomic Bulletin \& Review, 14, 699-703.

Colom, R., Haier, R. J., Head, K., Álvarez-Linera, J., Quiroga, M. A., Shih, P. C., et al. (2009). Gray matter correlates of fluid, crystallized, and spatial intelligence: Testing the P-FIT model. Intelligence, 37, 124-135.

Conway, A. R. A., Cowan, N., \& Bunting, M. F. (2001). The cocktail party phenomenon revisited: The importance of working memory capacity. Psychonomic Bulletin and Review, 8, 331-335.

Conway, A. R. A., \& Engle, R. W. (1996). Individual differences in working memory capacity: More evidence for a general capacity theory. Memory, 4, 577-590.

Conway, A. R. A., \& Kane, M. J. (2001). Capacity, control and conflict: An individual differences perspective on attentional capture. In C. Folk \& B. Gibson (Eds.), Attraction, distraction and action: Multiple perspectives on attention capture (pp. 349-372). Amsterdam: Elsevier Science.

Corbetta, M., Patel, G. H., \& Shulman, G. L. (2008). The reorienting system of the human brain: From environment to theory of mind. Neuron, 58, 306-324.

Corbetta, M., \& Shulman, G. L. (2002). Control of goal-directed and stimulus-driven attention in the brain. Nature Reviews Neuroscience, 3, 215-229.

Coull, J. T., Jones, M. E. P., Egan, T. D., Frith, C. D., \& Maze, M. (2004). Attentional effects of noradrenaline vary with arousal level : Selective activation of thalamic pulvinar in humans. NeuroImage, $22,315-322$

Coyle, T. R. (2003). A review of the worst performance rule: Evidence, theory, and alternative hypotheses. Intelligence, 31, 567-587.

Critchley, H. D., Tang, J., Glaser, D., Butterworth, B., \& Dolan, R. J. (2005). Anterior cingulate activity during error and autonomic response. NeuroImage, 27, 885-895.

Cronbach, L. J. (1957). The two disciplines of scientific psychology. American Psychologist, 12, 671-684.

D’Ardenne, K., Eshel, N., Luka, J., Lenartowicz, A., Nystrom, L. E., \& Cohen, J. D. (2012). Role of prefrontal cortex and the midbrain dopamine system in working memory updating. Proceedings of the National Academy of Sciences, 109, 19900-19909.

Daneman, M., \& Carpenter, P. A. (1980). Individual differences in working memory and reading. Journal of Verbal Learning and Verbal Behavior, 19, 450-466.

Dosenbach, N. U., Fair, D. A., Cohen, A. L., Schlaggar, B. L., \& Petersen, S. E. (2008). A dual-networks architecture of top-down control. Trends Cognitive Science, 12, 99-105.

Drew, T., \& Vogel, E. K. (2008). Neural measures of individual differences in selecting and tracking multiple moving objects. Journal of Neuroscience, 28, 4183-4191.

Drummond, S. P. A., Bischoff-Grethe, Dinges, D. F., Ayalon, L., Mednick, S. C., \& Meloy, M. J. (2005). The neural basis of the psychomotor vigilance task. Sleep, 28, 1059-1068.

Duncan, J. (1995). Attention, intelligence, and the frontal lobes. In M. Gazzaniga (Ed.), The cognitive neurosciences (pp. 721-733). Cambridge: MIT Press.

Ebitz, R. B., \& Platt, M. L. (2015). Neuronal activity in primate dorsal anterior cingulate cortex signals task conflict and predicts adjustments in pupil-linked arousal. Neuron, 85, 628-640.

Eldar, E., Cohen, J. D., \& Niv, Y. (2013). The effects of neural gain on attention and learning. Nature Neuroscience, 16, 1146-1153.

Engle, R. W., \& Kane, M. J. (2004). Executive attention, working memory capacity, and a two- factor theory of cognitive control. In B. Ross (Ed.), The psychology of learning and motivation (Vol. 44, pp. 145-199). NY: Elsevier.

Engle, R. W., Tuholski, S. W., Laughlin, J. E., \& Conway, A. R. A. (1999). Working memory, short-term memory and general fluid intelligence: A latent-variable approach. Journal of Experimental Psychology: General, 128, 309-331.

Esterman, M., Noonan, S. K., Rosenberg, M., \& DeGutis, J. (2012). In the zone or zoning out? Tracking behavioral and neural fluctuations during sustained attention. Cerebral Cortex, 23, 2712-2723. 
Faraco, C. C., Unsworth, N., Langley, J., Terry, J. D., Li, K., Zhang, D., et al. (2011). Complex span tasks and hippocampal recruitment during working memory. Neurolmage, 55, 773-787.

Foote, S. L., Aston-Jones, G., \& Bloom, F. E. (1980). Impulse activity of locus coeruleus neurons in awake rats and monkeys is a function of sensory stimulation and arousal. Proceedings of the National Academy of Sciences, 77, 3033-3037.

Fox, M. D., Snyder, A. Z., Vincent, J. L., Corbetta, M., Van Essen, D. C., $\&$ Raichle, M. E. (2005). The human brain is intrinsically organized into dynamic, anticorrelated functional networks. Proceedings of the National Academy of Sciences, 102, 9673-9678.

Fox, K. C., Spreng, R. N., Ellamil, M., Andrews-Hanna, J. R., \& Christoff, K. (2015). The wandering brain: Meta-analysis of functional neuroimaging studies of mind-wandering and related spontaneous thought processes. NeuroImage, 111, 611-21.

Frank, M. J., Santamaria, A., O'Reilly, R., \& Willcutt, E. (2007). Testing computational models of dopamine and noradrenaline dysfunction in Attention Deficit/Hyperactivity Disorder. Neuropsychopharmacology, 32, 1583-1599.

Franklin, M. S., Broadway, J. M., Mrazek, M. D., Smallwood, J., \& Schooler, J. W. (2013). Window to the wandering mind: Pupillometry of spontaneous thought while reading. The Quarterly Journal of Experimental Psychology, 1-15.

Fried, M., Tsitsiashvili, E., Bonney, Y. S., Sterkin, A., Wygnanski-Jaffe, T., Epstein, T., et al. (2014). ADHD subjects fail to suppress eye blinks and mircosaccades while anticipating visual stimuli but recover with medication. Vision Research, 101, 62-72.

Fukuda, K., \& Vogel, E. K. (2009). Human variation in overriding attentional capture. Journal of Neuroscience, 29, 8726-8733.

Fukuda, K., \& Vogel, E. K. (2011). Individual differences in recovery time from attentional capture. Psychological Science, 22, 361-368.

Gabay, S., Pertzov, Y., \& Henik, A. (2011). Orienting of attention, pupil size, and the norepinephrine system. Attention, Perception \& Psychophysics, 73, 123-129.

Geva, R., Zivan, M., Warsha, A., \& Olchik, D. (2013). Alerting, orienting or executive attention networks: Differential patters of pupil dilations. Frontiers in Behavioral Neuroscience, 7, 145.

Gilzenrat, M. S., Nieuwenhuis, S., Jepma, M., \& Cohen, J. D. (2010). Pupil diameter tracks changes in control state predicted by the adaptive gain theory of locus coeruleus function. Cognitive, Affective, \& Behavioral Neuroscience, 10, 252-269.

Golchert, J., Smallwood, J., Jefferies, E., Seli, P., Huntenburg, J.M., Liem, F., Lauckner, M.E., Olgschlager, S., Bernhardt, B.C., Villringer, A., \& Margulies, D.S. (in press). Individual variation in intentionality in the mind-wandering state is reflected in the integration of the default-mode, fronto-parietal, and limbic networks. NeuroImage.

Goldinger, S. D., \& Papesh, M. H. (2012). Pupil dilation reflects the creation and retrieval of memories. Current Directions in Psychological Science, 21, 90-95.

Grandchamp, R., Braboszcz, C., \& Delorme, A. (2014). Occulometric variations during mind wandering. Frontiers in Psychology, 5, 31.

Gray, J. R., Chabris, C. F., \& Braver, T. S. (2003). Neural mechanisms of general fluid intelligence. Nature Neuroscience, 6, 316-322.

Grodsky, A., \& Giambra, L. M. (1990-1991). The consistency across vigilance and reading tasks of individual differences in the occurrence of task-unrelated and task-related images and thoughts. Imagination, Cognition and Personality 10, 39-52.

Gulbinaite, R., van Rijn, H., \& Cohen, M. X. (2014). Fronto-parietal network oscillations reveal relationship between working memory capacity and cognitive control. Frontiers in Human Neuroscience, 8, 761 .

Hasselmo, M. E., \& Sarter, M. (2011). Modes and models of forebrain cholinergic neuromodulation of cognition. Neuropsychopharmacology Reviews, 36, 52-73.
Heitz, R. P., \& Engle, R. W. (2007). Focusing the spotlight: Individual differences in visual attention control. Journal of Experimental Psychology: General, 136, 217-240.

Hess, E. H., \& Polt, J. M. (1964). Pupil size in relation to mental activity during simple problem- solving. Science, 143, 1190-1192.

Hockey, G. R. J. (1997). Compensatory control in the regulation of human performance under stress and high workload: A cognitiveenergetical framework. Biological Psychology, 45, 73-93.

Hopstaken, J. F., van deer Linden, D., Bakker, A. B., \& Kompier, M. A. J. (2015). A Multifaceted Investigation of the link between mental fatigue and task disengagement. Psychophysiology, 52, 305-315.

Hopstaken, J. F., van der Linden, D., Bakker, A. B., \& Komier, M. A. J. (2015). The window of my eyes: Task disengagement and mental fatigue covary with pupil dynamics. Biological Psychology, 110, $100-106$.

Hou, R. H., Freeman, C., Langley, R. W., Szabadi, E., \& Bradshaw, C. M. (2005). Does modafinil activate the locus coeruleus in man? Comparison of modafinil and clonidine on arousal and autonomic functions in human volunteers. Psychopharmacology, 181, 537-549.

Hutchison, K. A. (2011). The interactive effects of listwide control, item based control, and working memory capacity on Stroop performance. Journal of Experimental Psychology: Learning, Memory, and Cognition, 37, 851-860.

Imeraj, L., Sonuga-Barke, E., Antrop, I., Roeyers, H., Wiersema, R., Bal, S., et al. (2012). Altered circadian profiles in attention deficit/ hyperactivity disorder: An integrative review and theoretical framework for future studies. Neuroscience \& Biobehavioral Reviews, 36, 1897-1919.

Jepma, M., \& Nieuwenhuis, S. (2011). Pupil diameter predicts changes in the exploration-exploitation trade-off: Evidence for the adaptive gain theory. Journal of Cognitive Neuroscience, 23, 1587-1596.

Jodo, E., Chiang, C., \& Aston-Jones, G. (1998). Potent excitatory influence of prefrontal cortex activity on noradrenergic locus coeruleus neurons. Neuroscience, 83, 63-79.

Joshi, S., Li, Y., Kalwani, R. M., \& Gold, J. I. (2016). Relationship between pupil diameter and neuronal activity in the locus coeruleus, colliculi, and cingulate cortex. Neuron, 89, 221-234.

Jung, R. E., \& Haier, R. J. (2007). The parieto-frontal integration theory (P-FIT) of intelligence: Converging neuroimaging evidence. Behavioral and Brain Sciences, 30, 135-187.

Kahneman, D. (1973). Attention and effort. Upper Saddle River: Prentice Hall.

Kahneman, D., \& Beatty, J. (1966). Pupil diameter and load on memory. Science, 154, 1583-1585.

Kane, M. J., Bleckley, M. K., Conway, A. R. A., \& Engle, R. W. (2001). A controlled-attention view of working-memory capacity. Journal of Experimental Psychology: General, 130, 169-183.

Kane, M. J., Brown, L. E., Little, J. C., Silvia, P. J., Myin-Germeys, I., \& Kwapil, T. R. (2007). For whom the mind wanders, and when: An experience-sampling study of working memory and executive control in daily life. Psychological Science, 18, 614-621.

Kane, M.J., Conway, A.R.A., Hambrick, D.Z., \& Engle, R.W. (2007). Variation in working memory capacity as variation in executive attention and control. In A.R.A Conway, C.

Kane, M. J., \& Engle, R. W. (2002). The role of prefrontal cortex in working-memory capacity, executive attention, and general fluid intelligence: An individual differences perspective. Psychonomic Bulletin \& Review, 9, 637-671.

Kane, M. J., \& Engle, R. W. (2003). Working-memory capacity and the control of attention: The contributions of goal neglect, response competition, and task set to Stroop interference. Journal of Experimental Psychology: General, 132(1), 47-70.

Kane, M. J., Hambrick, D. Z., Tuholski, S. W., Wilhelm, O., Payne, T. W., \& Engle, R. W. (2004). The generality of working-memory capacity: A latent-variable approach to verbal and visuo-spatial memory 
span and reasoning. Journal of Experimental Psychology: General, 133, 189-217.

Kane, M. J., \& McVay, J. C. (2012). What mind-wandering reveals about executive-control abilities and failures. Current Directions in Psychological Science, 21, 348-354.

Kane, M. J., Meier, M. E., Smeekens, B. A., Gross, G. M., Chun, C. A., Silvia, P. J., et al. (2016). Individual differences in the executive control of attention, memory, and thought, and their associations with schizotypy. Journal of Experimental Psychology: General, 145, 1017-1048.

Kane, M. J., Poole, B. J., Tuholski, S. W., \& Engle, R. W. (2006). Working memory capacity and the top-down control of visual search: Exploring the boundaries of "executive attention.". Journal of Experimental Psychology: Learning, Memory, and Cognition, 32, 749-777.

Karatekin, C., Bingham, C., \& White, T. (2010). Oculomotor and pupillometric indices of pro- and antisaccade performance in youth-onset psychosis and attention deficit/hyperactivity disorder. Schizophrenia Bulletin, 36, 1167-1186.

Keller, J. B., Hedden, T., Thompson, T. W., Anteraper, S. A., Gabrieli, J. D., \& Whitfield-Gabrieli, S. (2015). Resting-state anticorrelations between medial and lateral prefrontal cortex: Association with working memory, aging, and individual differences. Cortex, 64, 271-280.

Kelly, A. M., Uddin, L. Q., Biswal, B. B., Castellanos, F. X., \& Milham, M. P. (2008). Competition between functional brain networks mediates behavioral variability. NeuroImage, 39, 527-537.

Keye, D., Wilhelm, O., Oberauer, K., \& van Ravenzwaaij, D. (2009). Individual differences in conflict-monitoring: Testing means and covariance hypothesis about the Simon and the Eriksen Flanker task. Psychological Research, 73, 762-776.

Killeen, P. R., Russell, V. A., \& Sergeant, J. A. (2013). A behavioral neuroenergetics theory of ADHD. Neuroscience \& Biobehavioral Reviews, 37, 625-657.

Klein, K., \& Boals, A. (2001). The relationship of life event stress and working memory capacity. Applied Cognitive Psychology, $15,565-579$

Kondo, H., Morishita, M., Osaka, N., Osaka, M., Fukuyama, H., \& Shibasaki, H. (2004). Functional roles of the cingulo-frontal network in performance on working memory. Neurolmage, 21, 2-14.

Konishi, M., McLaren, D. G., Engen, H., \& Smallwood, J. (2015). Shaped by the past: The default mode network supports cognition that is independent of immediate perceptual input. PLoS One, 10, e0132209.

Kristjansson, S. D., Stern, J. A., Brown, T. B., \& Rohrbaugh, J. W. (2009). Detecting phasic lapses of alterness using pupillometric measures. Applied Ergonomics, 40, 978-986.

Kyllonen, P. C., \& Christal, R. E. (1990). Reasoning ability is (little more than) working- memory capacity? Intelligence, 14, 389-433.

Kyllonen, P. C., \& Stephens, D. L. (1990). Cognitive abilities as determinants of success in acquiring logic skill. Learning and Individual Differences, 2, 129-160.

Laeng, B., Ørbo, M., Holmlund, T., \& Miozzo, M. (2011). Pupillary Stroop effects. Cognitive Processing, 12, 13-21.

Laeng, B., Sirois, S., \& Gredebäck, G. (2012). Pupillometry: A window to the preconscious? Perspectives on Psychological Science, 7, 18-27.

Larson, G. E., \& Alderton, D. L. (1990). Reaction time variability and intelligence: A "worst performance" analysis of individual differences. Intelligence, 14, 309-325.

Lenartowicz, A., Simpson, G. V., \& Cohen, M. S. (2013). Perspective: Causes and functional significance of temporal variations in attention control. Frontiers in Human Neuroscience, 7, 381.

Long, D. L., \& Prat, C. S. (2002). Working memory and Stroop interference: An individual differences investigation. Memory \& Cognition, 30, 294-301.
Lowenstein, O., Feinberg, R., \& Lowenfeld, I. E. (1963). Pupillary movements during acute and chronic fatigue: A new test for the objective evaluation of tiredness. Investigative Ophthalmology, 2, 138-157.

Mason, M. F., Norton, M. I., Van Horn, J. D., Wegner, D. M., Grafton, S. T., \& Macrae, C. N. (2007). Wandering minds: The default network and stimulus-independent thought. Science, 315, 393-395.

Mather, M., \& Harley, C. (2016). The locus coeruleus: Essential for maintaining cognitive function and the aging brain. Trends in Cognitive Science, 20, 214-226.

McGaugh, J. L. (2006). Make mild moments memorable: Add a little arousal. Trends in Cognitive Sciences, 10, 345-347.

McLaren, J. W., Erie, J. C., \& Brubaker, R. F. (1992). Computerized analysis of pupillograms in studies of alertness. Investigative Ophthalmology \& Visual Sciences, 33, 671-676.

McNab, F., \& Klingberg, T. (2008). Prefrontal cortex and basal ganglia control access to working memory. Nature Neuroscience, 11, 103-107.

McVay, J. C., \& Kane, M. J. (2009). Conducting the train of thought: Working memory capacity, goal neglect, and mind wandering in an executive-control task. Journal of Experimental Psychology: Learning, Memory, and Cognition, 35, 196-204.

McVay, J. C., \& Kane, M. J. (2010). Does mind wandering reflect executive function or executive failure? Comment on Smallwood and Schooler (2006) and Watkins (2008). Psychological Bulletin, 136, 188-197.

McVay, J. C., \& Kane, M. J. (2012a). Why does working memory capacity predict variation in reading comprehension? On the influence of mind wandering and executive attention. Journal of Experimental Psychology: General, 141, 302-320.

McVay, J. C., \& Kane, M. J. (2012b). Drifting from slow to "D'oh!" Working memory capacity and mind wandering predict extreme reaction times and executive-control errors. Journal of Experimental Psychology: Learning, Memory, and Cognition, 38, $525-549$.

Meier, M. E., \& Kane, M. J. (2013). Working memory capacity and Stroop interference: Global versus local indices of executive control. Journal of Experimental Psychology: Learning, Memory, and Cognition, 39, 748-759.

Meier, M. E., \& Kane, M. J. (2015). Carving executive control at its joints: Working memory capacity predicts stimulus-stimulus, but not stimulus-response, conflict. Journal of Experimental Psychology: Learning, Memory, and Cognition, 41, 1849-1872.

Menon, V. (2011). Large-scale brain networks and psychopathology: A unifying triple-network model. Trends in Cognitive Sciences, 15, 483-506.

Menon, V., \& Uddin, L. Q. (2010). Saliency, switching, attention and control: A network model of insula function. Brain Structure and Function, 214, 655-667.

Millan, M. J. (2003). The neurobiology and control of anxious states. Progress in Neurobiology, 70, 83-244.

Miller, E. K., \& Cohen, J. D. (2001). An integrative theory of prefrontal cortex functioning. Annual Review of Neuroscience, 24, 167-202.

Miller, A. E., Watson, J. M., \& Strayer, D. L. (2012). Individual differences in working memory capacity predict action monitoring and the error-related negativity. Journal of Experimental Psychology: Learning, Memory, and Cognition, 38, 757-763.

Minamoto, T., Yaoi, K., Osaka, M., \& Osaka, N. (2015). The rostral prefrontal cortex underlies individual differences in working memory capacity: An approach from the hierarchical model of cognitive control. Cortex, 71, 277-290.

Minzenberg, M. J., Watrous, A. J., Yoon, J. H., Ursu, S., \& Carter, C. S. (2008). Modafinil shifts human locus coeruleus to low-tonic, high phasic activity during functional MRI. Science, 322, 1700-1702.

Minzenberg, M. J., Yoon, J. H., \& Carter, C. S. (2011). Modafinil modulation of the default mode network. Psychopharmacology, 215, $23-31$. 
Mittner, M., Boekel, W., Tucker, A., Turner, B. M., Heathcote, A., \& Forstmann, B. U. (2014). When the brain takes a break: A modelbased analysis of mind wandering. The Journal of Neuroscience, 34, 16286-16295.

Morad, Y., Lemberg, H., Yofe, N., \& Dagan, Y. (2000). Pupillography as an objective indicator of fatigue. Current Eye Research, 21, 535-542.

Morey, C. C., Elliott, E. M., Wiggers, J., Eaves, S. D., Shelton, J. T., \& Mall, J. T. (2012). Goal-neglect links Stroop interference with working memory capacity. Acta Psychologica, 141, 250-260.

Mrazek, M. D., Smallwood, J., Franklin, M. S., Chin, J. M., Baird, B., \& Schooler, J. W. (2012). The role of mind-wandering in measurements of general aptitude. Journal of Experimental Psychology: General, 788-798.

Murphy, P. R., O'Connell, R. G., O'Sullivan, M., Robertson, I. H., \& Balsters, J. H. (2014). Pupil diameter covaries with BOLD activity in human locus coeruleus. Human Brain Mapping, 35, 4140-4154.

Murphy, P. R., Robertson, I. H., Balsters, J. H., \& O'Connell, R. G. (2011). Pupillometry and P3 index the locus coeruleusnoradrenergic arousal function in humans. Psychophysiology, 48, $1532-43$.

Niendam, T. A., Laird, A. R., Ray, K. L., Dean, Y. M., Glahn, D. C., \& Carter, C. S. (2012). Meta- analytic evidence for a superordinate cognitive control network subserving diverse executive functions. Cognitive Affective Behavior in Neuroscience, 12, 241-68.

Osaka, M., Osaka, N., Kondo, H., Morishita, M., Fukuyama, H., Aso, T., et al. (2003). The neural basis of individual differences in working memory capacity: An fMRI study. Neurolmage, 18, 789-797.

Peavler, W. S. (1974). Pupil size, information overload, and performance differences. Psychophysiology, 11, 559-566.

Petersen, S. E., \& Posner, M. I. (2012). The attention system of the human brain: 20 years after. Annual Review of Neuroscience, 35, 73-89.

Phillips, M. A., Szabadi, E., \& Bradshaw, C. M. (2000). Comparison of the effects of clonidine and yohimbine on spontaneous pupillary fluctuations in healthy human volunteers. Psychopharmacology, 150, 85-89.

Poole, B. J., \& Kane, M. J. (2009). Working memory capacity predicts the executive control of visual search among distractors: The influence of sustained and selective attention. Quarterly Journal of Experimental Psychology, 62, 1430-1454.

Porter, G., Troscianko, T., \& Gilchrist, I. D. (2007). Effort during visual search and counting: Insights from pupillometry. Quarterly Journal of Experimental Psychology, 60, 211-229.

Raichle, M. E., MacLeod, A. M., Snyder, A. Z., Powers, W. J., Gusnard, D. A., \& Shulman, G. L. (2001). A default mode of brain function. Proceedings of the National Academy of Science, 98, 676-682.

Raizada, R. D. S., \& Poldrack, R. A. (2008). Challenge-driven attention: Interacting frontal and brainstem systems. Frontiers in Human Neuroscience, 1, 3.

Rajkowski, J., Kubiak, P., \& Aston-Jones, G. (1993). Correlations between locus coeruleus (LC) neural activity, pupil diameter and behavior in monkey support a role of LC in attention. Society for Neuroscience Abstracts, 19, 974.

Rajkowski, J., Lu, W., Zhu, Y., Cohen, J. D., \& Aston-Jones, G. (2000). Prominent projections from the anterior cingulate cortex to the locus coeruleus (LC) in rhesus monkey. Society for Neuroscience Abstracts, 26, 2230.

Ramos, B. P., \& Arnsten, A. F. (2007). Adrenergic pharmacology and cognition: Focus on the prefrontal cortex. Pharmacology \& Therapeutics, 113, 523-536.

Ratcliff, R., Schmiedek, F., \& McKoon, G. (2008). A diffusion model explanation of the worst performance rule fo rreaction time and IQ. Intelligence, 36, 10-17.

Redick, T. S. (2014). Cognitive control in context: Working memory capacity and proactive control. Acta Psychologica, 145, 1-9.
Redick, T. S., Calvo, A., Gay, C. E., \& Engle, R. W. (2011). Working memory capacity and go/no-go task performance: Selective effects of updating, maintenance, and inhibition. Journal of Experimental Psychology: Learning, Memory, and Cognition, 37, 308-324.

Redick, T. S., \& Engle, R. W. (2006). Working memory capacity and attention network test performance. Applied Cognitive Psychology, 20, 713-721.

Redick, T. S., \& Engle, R. W. (2011). Integrating working memory capacity and context-processing views of cognitive control. Quarterly Journal of Experimental Psychology, 64, 1048-1055.

Reimer, J., McGinley, M. J., Liu, Y., Rodenkirch, C., Wang, Q., McCormick, D. A., et al. (2016). Pupil fluctuations track rapid changes in adrenergic and cholinergic activity in cortex. Nature Communications, 7, 13289

Ressler, K. J., \& Nemeroff, C. B. (2001). Role of norepinephrine in the pathophysiology of neuropsychiatric disorders. CNS Spectrums, 6 , 663-670.

Richmond, L., Redick, T. S., \& Braver, T. (2015). Remembering to prepare: The benefits (and costs) associated with high working memory capacity. Journal of Experimental Psychology: Learning, Memory, and Cognition, 41, 1764-1777.

Ridderinkhof, K. R., \& Harsay, H. A. (2012). Cognitive control, cognitive search, and motivational salience: a systems neuroscience approach. In P. M. Todd, T. T. Hills, \& T. W. Robbins (Eds.), Cognitive search: Evolution, algorithms, and the brain (pp. 209-220). Cambridge: MIT Press.

Roberts, R. J., \& Pennington, B. F. (1996). An integrative framework for examining prefrontal cognitive processes. Developmental Neuropsychology, 12(1), 105-126.

Robertson, I. H. (2013). A noradrenergic theory of cognitive reserve: Implications for Alzheimer's disease. Neurobiology of Aging, 34, 298-308.

Robison, M.K., Gath, K.I., \& Unsworth, N. (in press). The neurotic wandering mind: An individual differences investigation of neuroticism, mind-wandering, and executive control. Quarterly Journal of Experimental Psychology.

Robison, M. K., \& Unsworth, N. (2015). Working memory capacity offers resistance to mind-wandering and external distraction in a context specific manner. Applied Cognitive Psychology, 29, 680-690.

Rosen, V. M., Bergeson, J. L., Putnam, K., Harwell, A., \& Sunderland, T. (2002). Working memory and apolipoprotein E: What's the connection? Neuropsycholgia, 40, 2226-2233.

Rowland, D. C., \& Kentros, C. G. (2008). Potential anatomical basis for attentional modulation of hippocampal neurons. Annals of the New York Academy of Sciences, 1129, 213-224.

Samuels, E. R., \& Szabadi, E. (2008a). Functional neuroanatomy of the noradrenergic locus coeruleus: Its roles in the regulation of arousal and autonomic function. Part I: Principles of functional organization. Current Neuropharmacology, 6, 235-253.

Samuels, E. R., \& Szabadi, E. (2008b). Functional neuroanatomy of the noradrenergic locus coeruleus: Its roles in the regulation of arousal and autonomic function. Part II: Physiological and pharmacological manipulations and pathological alterations of locus coeruleus activity in humans. Current Neuropharmacology, 6, 254-285.

Sara, S. J., \& Bouret, S. (2012). Orienting and reorienting: The locus coeruleus mediates cognition through arousal. Neuron, 76, 130-141.

Sarter, M., Gehring, W., \& Kozak, R. (2006). More attention must be paid: The neurobiology of attentional effort. Brain Research Reviews, 51, 145-160.

Sarter, M., \& Paolone, G. (2011). Deficits in attentional control: Cholinergic mechanisms and circuitry-based treatment approaches. Behavioral Neuroscience, 125, 825-835.

Schamader, T., \& Johns, M. (2003). Converging evidence that stereotype threat reduces working memory capacity. Journal of Personality and Social Psychology, 85, 440-452. 
Schmiedek, F., Oberauer, K., Wilhelm, O., Süß, H. M., \& Wittmann, W. W. (2007). Individual differences in components of reaction time distributions and their relations to working memory and intelligence. Journal of Experimental Psychology: General, 136, 414-429.

Schneider, M., Hathway, P., Leuchs, L., Samann, P. G., Czisch, M., \& Spoormaker, V. I. (2016). Spontaneous pupil dilations during the resting state are associated with activation of the salience network. NeuroImage, 139, 189-201.

Seeley, W. W., Menon, V., Schatzberg, A. F., Keller, J., Glover, G. H., Kenna, H., et al. (2007). Dissociable intrinsic connectivity networks for salience processing and executive control. Journal of Neuroscience, 27, 2349-2356.

Seli, P., Carriere, J. S. A., Thomson, D. R., Cheyne, J. A., Martens, K. A. E., \& Smilek, D. (2014). Restless mind, restless body. Journal of Experimental Psychology: Learning, Memory, and Cognition, 40, 660-668.

Seli, P., Cheyne, J. A., \& Smilek, D. (2013). Wandering minds and wavering rhythms: Linking mind wandering and behavioral variability. Journal of Experimental Psychology: Human Perception and Performance, 39, 1-5.

Seli, P., Risko, E. F., Smilek, D., \& Schacter, D. L. (2016). Mindwandering with and without intention. Trends in Cognitive Sciences, 20, 605-617.

Servan-Schreiber, D., Printz, H., \& Cohen, J. D. (1990). A network model of catecholamine effects: Gain, signal-to-noise ratio, and behavior. Science, 249, 892-895.

Smallwood, J., Brown, K. S., Mrazek, B., Baird, Franklin, M. S., \& Schooler, J. W. (2012). Insulation for daydreams: A role for tonic norepinephrine in the facilitation of internally guided thought. $P L O S$ One, 7, e33706.

Smallwood, J., \& Schooler, J. W. (2006). The restless mind. Psychological Bulletin, 132, 946-958.

Smallwood, J., Tipper, C., Brown, K., Baird, B., Engen, H., Michaels, J., et al. (2013). Escaping the here and now: Evidence for a role of the default mode network in perceptually decoupled thought. NeuroImage, 69, 120-125.

Smith, E. E., Geva, A., Jonides, J., Miller, A., Reuter-Lorenz, \& Koeppe, R. A. (2001). The neural basis of task-switching in working memory: Effects of performance and aging. Proceedings of the National Academy of Sciences, 98, 20095-2100.

Smith, A., \& Nutt, D. (1996). Noradrenaline and attention lapses. Nature, 380, 291.

Sonuga-Barke, E. J., \& Castellanos, F. X. (2007). Spontaneous attentional fluctuations in impaired states and pathological conditions: A neurobiological hypothesis. Neuroscience and Biobehavioral Reviews, 31, 977-986.

Spreng, R. N., DuPre, E., Selarka, D., Garcia, J., Gojkovic, S., Mildner, J., et al. (2014). Goal-congruent default network activity facilitates cognitive control. Journal of Neuroscience, 34, 14108-14114.

Sridharan, D., Levitin, D. J., \& Menon, V. (2008). A critical role for the right fronto-insular cortex in switching between central-executive and default-mode networks. Proceedings of the National Academy of Science, 105, 12569-12574.

Stawarczyk, D., Majerus, S., Maj, M., Van der Linden, M., \& D'Argembeau, A. (2011). Mind- wandering: Phenomenology and function as assessed with a novel experience sampling method. Acta Psychologica, 136, 370-381.

Szabadi, E. (2013). Functional neuroanatomy of the central noradrenergic system. Journal of Psychopharmacology, 27, 659-693.

Todd, J. J., \& Marois, R. (2004). Capacity limit of visual short-term memory in human posterior parietal cortex. Nature, 428, 751-754.

Todd, J. J., \& Marois, R. (2005). Posterior parietal cortex activity predicts individual differences in visual short-term memory capacity. Cognitive, Affective, and Behavioral Neuroscience, 5, 144-155.
Tsukahara, J. S., Harrison, T. L., \& Engle, R. W. (2016). The relationship between baseline pupil size and intelligence. Cognitive Psychology, 91, 109-123.

Ullsperger, M., Harsay, H. A., Wessel, J. R., \& Ridderinkhof, K. R. (2010). Conscious perception of errors and its relation to the anterior insula. Brain Structure and Function, 214, 629-643.

Underwood, B. J. (1975). Individual differences as a crucible in theory construction. American Psychologist, 30, 128-134.

Unsworth, N. (2009). Variation in working memory capacity, fluid intelligence, and episodic recall: A latent variable examination of differences in the dynamics of free recall. Memory \& Cognition, 37, 837-849.

Unsworth, N. (2015). Consistency of attentional control as an important cognitive trait: A latent variable analysis. Intelligence, 49, 110-128.

Unsworth, N. (2016). The many facets of individual differences in working memory capacity. In B. Ross (Ed.). The psychology of learning and motivation 65, 1-46.

Unsworth, N., Brewer, G. A., \& Spillers, G. J. (2012). Variation in cognitive failures: An individual differences investigation of everyday attention and memory failures. Journal of Memory \& Language, 67, 1-16.

Unsworth, N., \& Engle, R. W. (2005). Individual differences in working memory capacity and learning: Evidence from the serial reaction time task. Memory \& Cognition, 33, 213-220.

Unsworth, N., \& Engle, R. W. (2007). The nature of individual differences in working memory capacity: Active maintenance in primary memory and controlled search from secondary memory. Psychological Review, 114, 104-132.

Unsworth, N., Fukuda, K., Awh, E., \& Vogel, E. K. (2014). Working memory and fluid intelligence: Capacity, attention control, and secondary memory retrieval. Cognitive Psychology, 71, 1-26.

Unsworth, N., Fukuda, K., Awh, E., \& Vogel, E. K. (2015). Working memory delay activity predicts individual differences in cognitive abilities. Journal of Cognitive Neuroscience, 27, 853-865.

Unsworth, N., \& McMillan, B. D. (2013). Mind wandering and reading comprehension: Examining the roles of working memory capacity, interest, motivation, and topic experience. Journal of Experimental Psychology: Learning, Memory, \& Cognition, 39, 832-842.

Unsworth, N., \& McMillan, B. D. (2014a). Similarities and differences between mind-wandering and external distraction: A latent variable analysis of lapses of attention and their relation to cognitive abilities. Acta Psychologica, 150, 14-25.

Unsworth, N., \& McMillan, B. D. (2014b). Fluctuations in pre-trial attentional state and their influence on goal neglect. Consciousness and Cognition, 26, 90-96.

Unsworth, N., McMillan, B. D., Brewer, G. A., \& Spillers, G. J. (2012). Everyday attention failures: An individual differences investigation. Journal of Experimental Psychology: Learning, Memory, \& Cognition, 38, 1765-1772.

Unsworth, N., Miller, J. D., Lakey, C. E., Young, D. L., Meeks, J. T., Campbell, W. K., et al. (2009). Exploring the relations among executive functions, fluid intelligence, and personality. Journal of Individual Differences, 30, 194-200.

Unsworth, N., Redick, T. S., Lakey, C. E., \& Young, D. L. (2010). Lapses in sustained attention and their relation to executive and fluid abilities: An individual differences investigation. Intelligence, 38, 111-122.

Unsworth, N., Redick, T. S., Spillers, G. J., \& Brewer, G. A. (2012). Variation in working memory capacity and cognitive control: Goal maintenance and micro-adjustments of control. Quarterly Journal of Experimental Psycholog, 65, 326-355.

Unsworth, N., \& Robison, M. K. (2015). Individual differences in the allocation of attention to items in working memory: Evidence from pupillometry. Psychonomic Bulletin \& Review, 22, 757-765.

Unsworth, N., \& Robison, M. K. (2016a). The influence of lapses of attention on working memory capacity. Memory \& Cognition, 44, 188-196. 
Unsworth, N., \& Robison, M. K. (2016b). Pupillary correlates of lapses of sustained attention. Cognitive, Affective, \& Behavioral Neuroscience, 16, 601-615.

Unsworth, N., \& Robison, M.K. (2016b). The importance of arousal for variation in working memory capacity and attention control: A latent variable pupillometry study. Manuscript submitted for publication.

Unsworth, N., Schrock, J. C., \& Engle, R. W. (2004). Working memory capacity and the antisaccade task: Individual differences in voluntary saccade control. Journal of Experimental Psychology: Learning, Memory, and Cognition, 30, 1302-1321.

Unsworth, N., \& Spillers, G. J. (2010). Working memory capacity: Attention, memory, or both? a direct test of the dual-component model. Journal of Memory and Language, 62, 392-406.

Usher, M., Cohen, J. D., Servan-Schreiber, D., Rajkowski, J., \& AstonJones, G. (1999). The role of locus coeruleus in the regulation of cognitive performance. Science, 283, 549-554.

Usher, M., \& Davelaar, E. J. (2002). Neuromodulation of decision and response selection. Neural Networks, 15, 635-645.

van den Brink, R. L., Murphy, P. R., \& Nieuwenhuis, S. (2016). Pupil diameter tracks lapses of attention. PLOS ONE, 11, e0165274.

van Steenbergen, H., \& Band, G. P. H. (2013). Pupil dilation in the Simon task as a marker of conflict processing. Frontiers in Human Neuroscience, 7, 215.

van Steenbergen, H., Band, G. P. H., \& Hommel, B. (2015). Does conflict help or hurt cognitive control? Initial evidence for an inverted Ushape relationship between perceived task difficulty and conflict adaptation. Frontiers in Psychology, 6, 974

Varazzani, C., San-Galli, A., Dilardeau, S., \& Bouret, S. (2015). Noradrenaline and dopamine neurons in the reward/effort tradeoff: A direct electrophysiological comparison in behaving monkeys. Journal of Neuroscience, 35, 7866-7877.

Vatansever, D., Menon, D. K., Manktelow, A. E., \& Sahakian, B. J. (2015). Default mode dynamics for global functioning integration. Journal of Neuroscience, 35, 15254-15262.

Vazey, E.M., \& Aston-Jones, G. (2012). The emerging role of norepinephrine in cognitive dysfunction of Parkinson's disease. Frontiers in Behavioral Neuroscience 6, 48.

Vincent, J. L., Kahn, I., Snyder, A. Z., Raichle, M. E., \& Buckner, R. L. (2008). Evidence for a frontoparietal control system revealed by intrinsic functional connectivity. Journal of Neurophysiology, 100, $3328-3342$
Vogel, E. K., \& Machizawa, M. G. (2004). Neural activity predicts individual differences in visual working memory capacity. Nature, 428 , 784-751

Vogel, E. K., McCollough, A. W., \& Machizawa, M. G. (2005). Neural measures reveal individual differences in controlling access to visual working memory. Nature, 438, 500-503.

Wang, C., Brien, D. C., \& Munoz, D. P. (2015). Pupil size reveals preparatory processes in the generation of pro-saccades and anti-saccades. European Journal of Neuroscience, 41, 1102-1110.

Weissman, D. H., Roberts, K. C., Visscher, K. M., \& Woldorff, M. G. (2006). The neural bases of momentary lapses of attention. Nature Neuroscience, 9, 971-978.

Weldon, R. B., Mushlin, H., Kim, B., \& Sohn, M. H. (2013). The effect of working memory capacity on conflict monitoring. Acta Psychologica, 142, 6-14.

Wessel, J. R., Danielmeier, C., \& Ullsperger, M. (2011). Error awareness revisited: Accumulation of multimodal evidence from central and autonomic nervous systems. Journal of Cognitive Neuroscience, 23, 3021-3036.

Westbrook, A., \& Braver, T. S. (2016). Dopamine does double duty in motivating cognitive effort. Neuron, 89, 695-710.

Wilhelm, B., Giedke, H., Ludtke, H., Bittner, E., Hofmann, A., \& Wilhelm, H. (2001). Daytime variations in central nervous system activation measured by a pupillographic sleepiness test. Journal of Sleep Research, 10, 1-7.

Wright, T. J., Boot, W. R., \& Morgan, C. S. (2013). Pupillary response predicts multiple object tracking load, error rate, and conscientiousness, but not inattentional blindness. Acta Psychologica, 144, 6-11.

Yellin, D., Berkovich-Ohana, A., \& Malach, R. (2015). Coupling between pupil fluctuations and resting-state fMRI uncovers a slow build-up of antagonistic responses in the human cortex. NeuroImage, 106, 414-427.

Yerkes, R. M., \& Dodson, J. D. (1908). The relation of strength of stimulus to rapidity of habit- formation. Journal of Comparative Neurology and Psychology, 18, 459-482.

Zénon, A., Sidibé, M., \& Olivier, E. (2014). Pupil size variations correlate with physical effort perception. Frontiers in Behavioral Neuroscience, 8, 286.

Zylberberg, A., Oliva, M., \& Sigman, M. (2012). Pupil dilation: a fingerprint of temporal selection during the "attentional blink". Frontiers in Psychology, 3, 316. 